\title{
Spectrum Trading in Cognitive Radio Networks: A Contract-Theoretic Modeling Approach
}

\author{
Lin Gao, Xinbing Wang, Member, IEEE, Youyun Xu, Member, IEEE, and Qian Zhang, Senior Member, IEEE
}

\begin{abstract}
Cognitive radio is a promising paradigm to achieve efficient utilization of spectrum resource by allowing the unlicensed users (i.e., secondary users, SUs) to access the licensed spectrum. Market-driven spectrum trading is an efficient way to achieve dynamic spectrum accessing/sharing. In this paper, we consider the problem of spectrum trading with single primary spectrum owner (or primary user, PO) selling his idle spectrum to multiple SUs. We model the trading process as a monopoly market, in which the $P O$ acts as monopolist who sets the qualities and prices for the spectrum he sells, and the SUs act as consumers who choose the spectrum with appropriate quality and price for purchasing. We design a monopolist-dominated quality-price contract, which is offered by the $P O$ and contains a set of qualityprice combinations each intended for a consumer type. A contract is feasible if it is incentive compatible (IC) and individually rational (IR) for each SU to purchase the spectrum with the quality-price intended for his type. We propose the necessary and sufficient conditions for the contract to be feasible. We further derive the optimal contract, which is feasible and maximizes the utility of the PO, for both discrete-consumer-type model and continuous-consumer-type model. Moreover, we analyze the social surplus, i.e., the aggregate utility of both PO and SUs, and we find that, depending on the distribution of consumer types, the social surplus under the optimal contract may be less than or close to the maximum social surplus.
\end{abstract}

Index Terms-Cognitive Radio, Spectrum Trading, Contract Theory, Quality Discrimination

\section{INTRODUCTION}

C URRENTLY, wireless communication networks suffer from the scarcity in spectrum resource and inefficiency in spectrum usage. Cognitive radio has been viewed as a novel approach for improving the utilization of spectrum resource. Cognitive radio networks are designed based on the concept of dynamic spectrum sharing where cognitive radio users can opportunistically share the radio spectrum. For example, in a vertical spectrum sharing model, a primary spectrum owner (PO) can share (or sell) his licensed spectrum with (to) the unlicensed users (i.e., secondary users, SUs). To realize this, it is essential to design a spectrum sharing mechanism in which the PO has incentive to lease his spectrum to SUs and the SUs also have incentive to employ the spectrum from the PO.

While most of the researchers emphasized the technical aspect of spectrum sharing (e.g., spectrum sensing in physical layer, dynamic spectrum access protocol in MAC layer, etc.), in this paper we focus on the economic aspect of spectrum

Manuscript received 1 December 2009; revised 1 July 2010.

L. Gao, X. Wang and Y. Xu are with the Department of Electronic Engineering, Shanghai Jiao Tong University, Shanghai, China (email: \{gaull, xwang8, xuyouyun\}@sjtu.edu.cn).

Q. Zhang is with the Department of Department of Computer Science and Engineering, Hong Kong University of Science and Technology, Hongkong (email: qianzh@cse.ust.hk).

Digital Object Identifier 10.1109/JSAC.2011.110415. sharing. Specifically, we consider the issue of spectrum trading between single PO and multiple SUs in a cognitive radio environment, where the term "spectrum trading" refers to the processes of selling and buying spectrum resource between PO and SUs, and we focus on the attribute of spectrum trading through the notion of "quality". Unlike the existing literature, we associate the trading goods (i.e., spectrum resource) with an attribute: quality, that is, each spectrum resource can be traded in different qualities. Moreover, we classify the SUs into multiple categories (types) according to their preference for a given spectrum quality. We refer to this as quality discrimination spectrum trading.

We model the trading process as a monopoly market, in which the PO acts as monopolist who sets the qualities and prices for the spectrum he sells, and the SUs act as consumers who choose the spectrum with appropriate quality and price for purchasing. The aim of this paper is to design a spectrum trading mechanism which maximizes the utility (revenue) of PO and meanwhile enhances the satisfactions of SUs. For this purpose, we introduce the concept of contract in economics, a widely used mechanism in supply chain designing in economics which provides incentives to all of the members in the chain so that the decentralized supply chain behaves nearly or exactly the same as the integrated one.

The main contributions of the article are as follows:

- We first consider the issue of quality discrimination for the spectrum trading with multiple consumer types, from which the PO can gain more revenue than traditional spectrum trading schemes such as auction, pricing, etc.

- We introduce the concept of contract into the quality discrimination spectrum trading, and design a monopolistdominated quality-price contract, which is offered by the $\mathrm{PO}$ and contains a set of quality-price combinations each intended for a consumer type.

- We propose the necessary and sufficient conditions for the feasible contract, which is incentive compatible (IC) and individually rational (IR) for each SU to purchase the spectrum with the quality-price intended for his type. We further derive the optimal contract which is feasible and maximizes the revenue of the PO. We find that the feasible contract can naturally reduce the interference between the primary network and secondary network.

- We analyze the social surplus, i.e., the aggregate utility of both PO and SUs, and we find that, depending on the distribution of consumer types, the social surplus under the optimal contract may be less than or close to the maximum social surplus.

The rest of this paper is organized as follows. In Section II, we review the related work. In Section III we provide the 
system model and contract formulation. In Section IV and Section V, we propose the optimal contracts for discreteconsumer-type model and continuous-consumer-type model, respectively. In Section VI, we present the simulation results and analyze the social surplus. Finally, we conclude our work in Section VII.

\section{RELATED WORK}

\section{A. Cognitive Radio and Dynamic Spectrum Access}

There are several comprehensive surveys on cognitive radio techniques [1], different spectrum sharing models [2], and challenges and issues in designing dynamic spectrum access networks [3], respectively. In cognitive radio networks, spectrum management is an important functionality that involves dynamic spectrum accessing/sharing and pricing, with an aim to satisfying the requirements of both primary and secondary users. In [4], Hwang et al. examined strategic approaches in the service providers', manufacturers', and policy makers' standpoints for the successful commercialization of cognitive radio with the dynamic spectrum management policy. In [5], an optimization problem was formulated for spectrum access to obtain the solution that gives the highest utility to the cognitive radio users. In [6], a game-theoretic adaptive channel allocation scheme was proposed for cognitive radio networks. In [7], a two tier dynamic spectrum allocation system was analyzed for non-strategic users who obtain spectrum from multiple SPs. In [8], a hybrid game approach which contains both cooperative behavior and competitive behavior was proposed for the cognitive radio networks with multi-hop communication links. In [9], Grokop et al. studied spectrum sharing between wireless devices operating under a random access protocol. Further, Liu et al. proposed some special applications of spectrum sensing/accessing such as localization [10][11] and monitoring [12]. However, the above works focused on the technical aspect of dynamic spectrum accessing/sharing, without considering the incentive issue (e.g., pricing) and economic aspect in spectrum sharing.

\section{B. Spectrum Trading in Cognitive Radio Networks}

Recently, many researchers paid their attention to the economic aspect of dynamic spectrum sharing, which is also referred to as spectrum trading. In [13], Niyato et al. discussed the concept of spectrum trading in the context of different spectrum sharing models, and outlined different forms of spectrum trading, the related research problems, and the different solution approaches. In [14], Niyato et al. studied the spectrum trading with multiple POs selling spectrum opportunities to multiple SUs. The evolution and the dynamic behavior of SUs was modeled as an evolutionary game, and the competition among the PUs was modeled as a noncooperative game. In spectrum trading, pricing is a major issue that determines the value (or worth) of the spectrum to the spectrum seller and buyer. In [15], an integrated pricing, allocating, and billing system was proposed for cognitive radio networks. In [16], a joint power/channel allocation scheme was proposed that used a distributed pricing strategy to improve the network's performance. In [17], a non-cooperative game based pricing scheme was proposed for uplink power control in cognitive radio networks. Besides, market-driven auction is an effective mechanism to allocate resources and determine trading prices. In [18], a bandwidth auction model was proposed for the problem of dynamic spectrum sharing. In [19], an auction mechanism was applied to the problem of spectrum sharing among users using spread spectrum signaling to access the channel. In [20], a progressive auction mechanism was proposed for dynamic spectrum sharing between multiple spectrum sellers and buyers.

Another issue in spectrum trading is competition/cooperation among cognitive radio entities involved in spectrum trading (i.e., spectrum sellers and buyers). In [21], Xing et al. considered competition among multiple primary users who sell radio spectrum to the secondary users. In [22], Ileri et al. considered competition among multiple secondary users who access the radio spectrum owned by the primary users in a cognitive radio environment. In [23], Gandhi et al. proposed a framework of spectrum trading based on an auction mechanism for dynamic spectrum access. However, the above works did not consider the heterogeneity in consumer (buyer) type, and the spectrum resource was traded in the same quality with different buyers, that is, they ignored the issue of quality discrimination for the spectrum trading.

\section{Contract in Supply Chain}

Supply chain contracts have been studied extensively in economics, operations management, and marketing science literatures (see [24] and [25] for recent surveys). Supply chain contract can be viewed as a mechanism to coordinate production (either quantity or quality) and pricing, so that the decentralized supply chain behaves nearly or exactly the same as the integrated one. In [26], Corbet et al. used the bilateral monopoly setting to analyze three contracts under full and incomplete information in the setting of deterministic demand. In [27], Ha studied optimal contracts in a more general setting than Corbet, including nonlinear stochastic demand, at the expense of gaining less insight into the value of information and of more general contracts. In [28], Weng studied quantity discounts for achieving coordination. In [29], Chen et al. extended this to multiple customers and more general cost structures. In this paper, we introduce the concept of contract into cognitive radio environment, and design a quality-price contract for the quality discrimination spectrum trading.

\section{System Model And PRoblem Formulation}

\section{A. PO Model}

We consider the cognitive radio network including a primary network and a secondary network. The primary network consists of a primary spectrum owner (PO) and a set of subscribed primary users (PUs). The PO can be a base station or an access point, and servers the subscribed PUs. The spectrum possessed by the PO is under-utilized, that is, there may exist some idle spectrum bands which are not used by the PUs at a particular time. For simplicity, we refer to such idle spectrum bands as residual bands or residual channels (or channels for short). The secondary network consists of a set of secondary transmitter and receiver pairs, where each secondary transmitter always has some packets to exchange 
with the intended receiver. We refer to a pair of secondary transmitter and receiver as a secondary link or secondary user (SU). Therefore, the PO is willing to sell his residual channels to the SUs for enhanced profit, and the SUs are also willing to purchase channels for their interested services. An example of cognitive radio network with single PO and 5 SUs can be seen in Figure 1.

Since the PO dominates the trading process, we model the spectrum trading as a monopoly market, in which the PO acts as the monopolist and the SUs act as the consumers. The monopolist sets the qualities and prices for his products (residual channels) relatively. A set of all qualities is called $\Omega$ and a set of all prices is called $\Pi$. Note that each quality $q \in \Omega$ corresponds to a price $\pi \in \Pi$. For simplicity, we denote the price corresponding to quality $q$ as $\pi(q)$. The consumers decide whether to buy a channel and which qualities they are going to buy, given the $\Omega$ and $\Pi$ offered by the PO. Since each channel can be traded in different qualities, we refer to this as quality discrimination spectrum trading.

In our model, we define the quality of a channel as the maximum allowable power the SUs can transmit on the channel. In other words, if an SU purchases a channel with quality $q$, the $\mathrm{PO}$ will assign a residual channel to the $\mathrm{SU},{ }^{1}$ and the SU can transmit packets on the channel with power not more than $q$. Obviously the power level cannot be negative or infinity, i.e., $P_{\min } \leq q \leq P_{\max }$, where $P_{\min }$ and $P_{\max }$ are lower-bound and upper-bound of the maximal allowable transmitting power, respectively.

We define the cost of a channel (for the PO) as the expense of PO when SUs occupy and employ the channel. Such an expense consists of a fixed cost mainly including the leasing fee of channel license, and a quality-specific cost mainly including the performance degradation of primary service induced by the interfering of secondary transmission. ${ }^{2}$ Thus we can write the cost of a channel with quality $q$ as:

$$
C(q)=C_{0}+T(q)
$$

where $C_{0}>0$ is the fixed cost and $T(q)$ is the quality-specific cost. It is easy to see that $T(q)$ is non-negative and monotone increasing on $q$. We further assume that the marginal qualityspecific cost $T_{q}(q)$ is non-decreasing on $q$, that is, $T(q)$ grows more rapidly in high quality than it does in low quality. ${ }^{3}$ Thus we can easily find that $C_{q}(q)>0$ and $C_{q q}(q) \geq 0$.

We define the utility (or revenue) of PO by selling a channel with quality $q$ to SUs, denoted by $R(q)$, as the difference between the selling price and the cost of the channel, i.e.,

$$
R(q)=\pi(q)-C(q)
$$

The objective of PO is to maximize his utility. Obviously a rational $\mathrm{PO}$ would not like to accept negative utility from a channel, thus he will always set $\pi(q) \geq C(q), \forall q \in \Omega$.

\footnotetext{
${ }^{1}$ Besides, the PO will charge the payment $\pi(q)$ from the SU.

${ }^{2}$ Such an interference may be caused by the imperfect orthogonality of channels (e.g., CDMA-based channels), side lobes effect in frequency (e.g., FDMA-based channels) or time dispersion caused by multipath propagation delays (e.g., TDMA-based channels).

${ }^{3}$ For simplicity, we write $\partial f(.) / \partial x$ as $f_{x}($.$) if f($.$) is continuously differ-$ entiable with respect to $x$. Similarly, we write $\partial^{2} f(.) / \partial x^{2}$ and $\partial^{2} f(.) / \partial x \partial y$ as $f_{x x}($.$) and f_{x y}($.$) , respectively, and so on.$
}

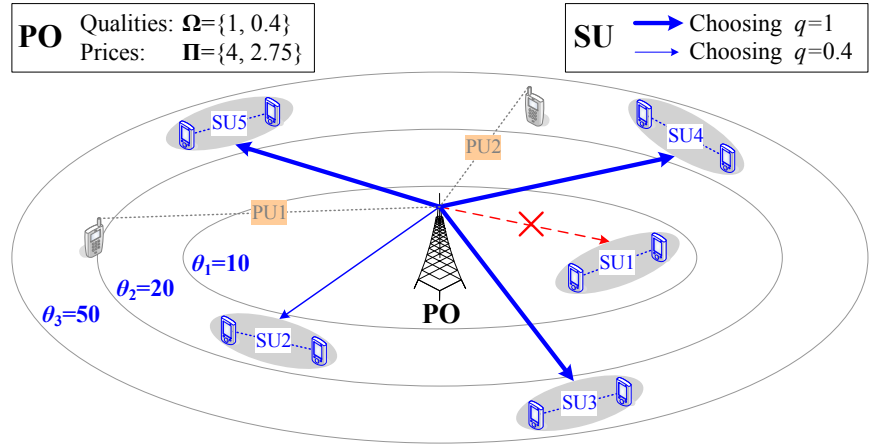

Fig. 1. An example of cognitive radio network with single PO, 2 PUs and 5 SUs.

Furthermore, to facilitate the describing, we define a quality $q=\mathrm{Na}$ to denote an aborted trading process, that is, the $\mathrm{SU}$ will not buy any channel if he chooses the quality $q=\mathrm{Na}$, equivalently the PO will not sell channels to the SUs who choose the quality $q=\mathrm{Na}$. Obviously in this case, the SUs do not need to pay for the channels and the PO will not suffer from any expense, i.e., $\pi(\mathrm{Na})=C(\mathrm{Na})=0$. Note that the quality $q=\mathrm{Na}$ is impliedly contained in $\Omega$, since not only the PO can decide whether to sell his channels, the SUs can also decide whether to buy channels from PO.

\section{B. SU Model}

We assume that each SU prefers higher channel capacity, which is not only related to the channel quality (i.e., the allowable transmitting power), but also related to the path loss factor between secondary transmitter and receiver, and the interference come from primary network. Specifically, for a given transmitting power $q$, the channel capacity for $\mathrm{SU} i$ can be given by Shannon-Hartley theorem:

$$
\Phi(q)=W \log _{2}\left(1+q \cdot \frac{L_{i}}{I_{i}+J_{i}+\sigma^{2}}\right)
$$

where $W$ is the channel bandwidth, $\sigma^{2}$ is the noise variance, $L_{i}$ is the path loss factor between the transmitter and receiver of SU $i, I_{i}$ and $J_{i}$ are respectively the interference come from the transmission of PO and PUs. Without loss of generality, we assume $W=1$ and $\sigma^{2}$ is identical for all SUs.

According to the difference in channel capacity for a given channel quality, we classify the SUs into different categories (types). Thus we can use the expression $\frac{L_{i}}{I_{i}+J_{i}+\sigma^{2}}$ to denote the consumer type. Specifically, we refer to an SU $i$ as a type- $\theta$ SU if $\frac{L_{i}}{I_{i}+J_{i}+\sigma^{2}}=\theta$. Accordingly, for a given channel quality $q$, the channel capacity for a type- $\theta$ SU can be written as $\Phi(q)=\log _{2}(1+q \cdot \theta)$. Obviously the consumer type is increasing with the path loss term $L_{i}$ and decreasing with the interference terms $I_{i}$ and $J_{i}$. We denote the set of all consumer types as $\Theta$, which can be a discrete set or a continuous region.

The SU can obtain his own type by measuring the wireless environment, while the PO cannot know the exact type of a particular SU, that is, the consumer type is private information for each SU. Nevertheless, we assume the PO has some statistical information about the consumer types, e.g., the probability distribution of consumer type.

We define the valuation of a type- $\theta$ SU for a channel with quality $q$, denoted by $V(\theta, q)$, as the benefit by employing 
the channel, which can be formulated as a strictly monotone increasing function on channel capacity. Without loss of generality, we define $V(\theta, q)$ as a linear function on capacity, i.e.,

$$
V(\theta, q) \triangleq w \log _{2}(1+q \cdot \theta)
$$

where $w>0$ is the equivalent valuation per unit channel capacity contributes to the overall valuation, which is a predefined parameter and identical for all SUs. For simplicity, we assume $w=1$ in the rest of the paper. Note that Eq. (3) is only available as $q \in\left[P_{\min }, P_{\max }\right]$. In the case of $q=\mathrm{Na}$, we define $V(\theta, \mathrm{Na})=0$. Furthermore, we define the following preference order on quality: for $\forall q \in\left[P_{\min }, P_{\max }\right]$, we have $q>\mathrm{Na}$. Obviously such a definition coincides with the nature of quality, i.e., $C(q)>C(\mathrm{Na})$ and $V(\theta, q)>V(\theta, \mathrm{Na})$.

It is easy to see that $V_{q}(\theta, q)>0$ and $V_{\theta}(\theta, q)>0$ for all $\forall q>\mathrm{Na}$, that is, every SU prefers higher channel quality, and for a given quality, a higher type SU has greater valuation than a lower one. We can further see that $V_{q q}(\theta, q)<0$, that is, $V(\theta, q)$ grows more slowly in a higher quality.

We define the utility of a type- $\theta$ SU by purchasing a channel with quality $q$, denoted by $U(\theta, q)$, as the difference between his valuation for the channel and the price of the channel, i.e.,

$$
U(\theta, q)=V(\theta, q)-\pi(q)
$$

We assume that each SU is selfish and rational, whose objective is always to maximize his utility, thus the optimal strategy for a type- $\theta$ SU can be written as:

$$
\mathcal{B}(\theta)=\arg \max _{q \in \Omega} U(\theta, q)
$$

Note that $\mathcal{B}(\theta)=\mathrm{Na}$ denotes that the optimal strategy for a type- $\theta \mathrm{SU}$ is to purchase nothing.

Figure 1 presents an example of cognitive radio network with single PO, 2 PUs and 5 SUs. As we assume $L_{i}$ and $J_{i}$ are approximately the same for all SUs, the type of SU $i$ is determined solely by the interference come from the PO, i.e., $I_{i}$, which is mainly related to the distance between the SU and PO. We assume that the SUs within the same circular ring have nearly the same $I_{i}$, thus we can divide the SUs into 3 types: $\theta_{1}, \theta_{2}$ and $\theta_{3}$, where SU1 is in type- $\theta_{1}$, SU2 is in type- $\theta_{2}$ and SU3 SU5 are in type- $\theta_{3}$. The qualities the PO selected are $\Omega=\{1,0.4\}$, and the relative prices are $\Pi=\{4,2.75\}$. Provided that $\theta_{1}=10, \theta_{2}=20$ and $\theta_{3}=50$, the type- $\theta_{3}$ SUs would like to purchase the channel with quality $q=1$ since $U\left(\theta_{3}, 1\right)=\log _{2}(1+50)-4>U\left(\theta_{3}, 0.4\right)=\log _{2}(1+$ $20)-2.75$, the type- $\theta_{2}$ SUs would like to purchase the channel with quality $q=0.4$ since $U\left(\theta_{2}, 1\right)=\log _{2}(1+20)-4<$ $U\left(\theta_{2}, 0.5\right)=\log _{2}(1+8)-2.75$, and the type- $\theta_{L}$ SUs will not purchase anything since $U\left(\theta_{1}, q\right)<0$ for both $q \in \Omega$, that is, we have $\mathcal{B}\left(\theta_{3}\right)=1, \mathcal{B}\left(\theta_{2}\right)=0.4$ and $\mathcal{B}\left(\theta_{1}\right)=\mathrm{Na}$.

\section{Social surplus}

We define the social surplus generated by PO's selling of a channel with quality $q$ to a type- $\theta$ SU, denoted by $S(\theta, q)$, as the aggregate utilities of both PO and SU, i.e.,

$$
S(\theta, q) \triangleq R(q)+U(\theta, q)=V(\theta, q)-C(q)
$$

where the price $\pi(q)$ in both utilities cancels each other out.

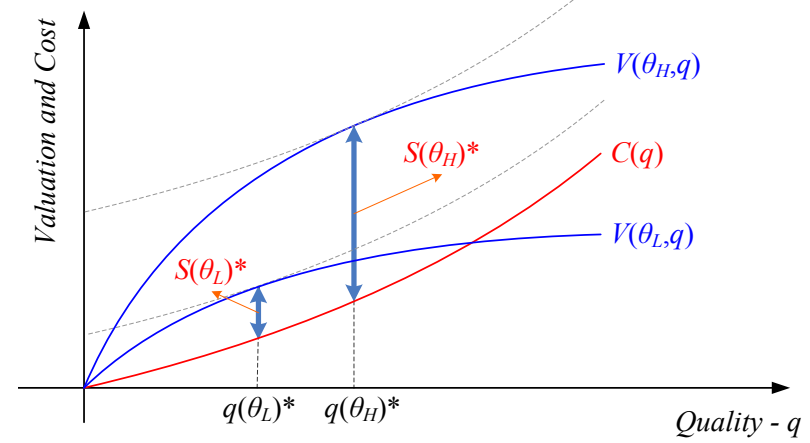

Fig. 2. An illustration of $q(\theta)^{*}$ and $S(\theta)^{*}$ for 2 consumer types.

Note that $S_{q q}(\theta, q)<0$ since $V_{q q}(\theta, q)<0$ and $C_{q q}(q) \geq$ 0 . Then, assuming the interior solutions, $q(\theta)^{*}$, the social optimal quality for type $\theta$, can be obtained by solving $V_{q}(\theta, q)-$ $C_{q}(q)=0$. Accordingly, the maximum social surplus for each type $\theta \in \Theta$ can be written as $S(\theta)^{*}=S\left(\theta, q(\theta)^{*}\right)$.

It is obvious that, for different consumer types, their social optimal qualities are different, so are their maximum social surplus. More precisely, the $q(\theta)^{*}$ and $S(\theta)^{*}$ are both monotone increasing with respect to $\theta$. In other words, it is socially desirable to offer a higher quality to the SUs with higher type. Figure 2 presents an illustration of the social optimal qualities and maximum social surplus for 2 consumer types $\theta_{H}$ and $\theta_{L}$ with $\theta_{H}>\theta_{L}$, from which we can easily find that $q\left(\theta_{H}\right)^{*}>q\left(\theta_{L}\right)^{*}$ and $S\left(\theta_{H}\right)^{*}>S\left(\theta_{L}\right)^{*}$.

However, in an open market, the social optimal qualities may not be adopted by both PO and SUs. In detail, the PO and SUs are selfish and rational, whose objectives are always to maximize their own utilities, without considering the social surplus. Nevertheless, we propose the social optimal quality and maximum social surplus since it provides an upper bound of the aggregate utility of PO and SUs.

\section{Contracts Formulation}

As mentioned previously, the monopolist's problem is to choose a set of qualities and a price for each quality, i.e., $\Omega$ and $\Pi$. Since only one quality will be chosen by each type of SUs, effectively the PO will be assigning a quality $q(\theta)$ and a price $\pi(q(\theta))$ to each consumer type $\theta \in \Theta$. For simplicity, we write $\pi(q(\theta))$ as $\pi(\theta)$ since $q(\theta)$ is a single value function. We refer to such a set of quality-price combinations as qualityprice contract, denoted by $\mathscr{C}=\{(q(\theta), \pi(\theta)) \mid \forall \theta \in \Theta\}$.

A feasible contract is a set of quality-price combinations such that for every type $\theta \in \Theta$, a type- $\theta$ consumer prefers the product with quality $q(\theta)$ at price $\pi(\theta)$ to any product with other quality and to not purchasing at all. In details, for the contract to be feasible in the open market, it must be incentive compatible (IC), that is, each SU finds it in his own interest to buy the product assigned his type. Formally, we can write the IC constraint as:

$$
V(\theta, q(\theta))-\pi(\theta) \geq V\left(\theta, q\left(\theta^{\prime}\right)\right)-\pi\left(\theta^{\prime}\right), \forall \theta^{\prime} \neq \theta
$$

for all type $\theta \in \Theta$.

Furthermore, as mentioned previously, a quality-price combination $(\mathrm{Na}, 0)$ is impliedly contained in any contract. An SU will choose $(\mathrm{Na}, 0)$ if his utility is negative under 
$(q(\theta), \pi(\theta)) .{ }^{4}$ We refer to this property as individual rationality (IR). Obviously, a feasible contract must be individual rational. Formally, we can write the IR constraint as:

$$
V(\theta, q(\theta))-\pi(\theta) \geq V(\theta, \mathrm{Na})-\pi(\mathrm{Na})=0
$$

for all type $\theta \in \Theta$.

In a word, a feasible contract must satisfy the IC and IR constraints, and any contract satisfying the IC and IR constraints must be feasible. For a feasible contract $\{(q(\theta), \pi(\theta))\}$, the overall utility of the PO can be written as:

$$
R=\sum_{\theta \in \Theta} N_{\theta}(\pi(\theta)-C(q(\theta)))
$$

where $N_{\theta}$ is the number of type- $\theta$ SUs. Note that Eq. (9) assumes the PO has a sufficient number of residual channels, i.e., greater than the number of SUs $\sum_{\theta \in \Theta} N_{\theta}$.

The optimal contract for the PO, denoted by $\{(\hat{q}(\theta), \hat{\pi}(\theta))\}$, is defined as a feasible contract which maximizes his utility. Formally, we can write the optimal contract as follows:

$$
\{(\hat{q}(\theta), \hat{\pi}(\theta))\}=\arg \max _{\substack{\{q(\theta)\} \\\{\pi(\theta)\}}} \sum_{\theta \in \Theta} N_{\theta}(\pi(\theta)-C(q(\theta)))
$$

subject to the IC and IR constraints in Eqs. (7) and (8).

So far, we have provided the contract formulation and the definition of optimal contract. We will address the following essential questions in the rest of this paper: (i) how to derive the optimal contract defined in Eq. (10) and (ii) whether this solution maximizes the social surplus.

\section{Optimal Contract in Discrete-Consumer-Type MODEL}

In discrete-consumer-type model, there is a finite number of consumer types, $T$, with indices $\theta_{1}, \theta_{2}, \ldots, \theta_{T}$. Without loss of generality, we assume that $\theta_{1}<\theta_{2}<\ldots<\theta_{T}$. For simplicity, we rewrite $N_{\theta_{t}}$, the number of type- $\theta_{t}$ consumers, as $N_{t}$. Similarly, we rewrite $q\left(\theta_{t}\right)$ and $\pi\left(\theta_{t}\right)$, the quality and price assigned to type- $\theta_{t}$ SUs, as $q_{t}$ and $\pi_{t}$, respectively. Suppose the PO has a sufficient number of channels, the optimal contract defined in Eq. (10) can be rewritten as:

$$
\left\{\left(\hat{q}_{t}, \hat{\pi}_{t}\right)\right\}=\arg \max _{\left\{q_{t}\right\}} \sum_{t=1}^{T} N_{t}\left(\pi_{t}-C\left(q_{t}\right)\right)
$$

subject to the IC and IR constraints in Eqs. (7) and (8).

We first present an essential property for SU's valuation: for a given quality increment, the valuation increment for a higher type $\mathrm{SU}$ is greater than that for a lower one. We refer to this as increasing preference property (IP). Formally, we can write this property as the following proposition.

Proposition 1 - (IP property): For any consumer types $\theta>$ $\theta^{\prime}$ and product qualities $q>q^{\prime}$, the following condition holds:

$$
V(\theta, q)-V\left(\theta, q^{\prime}\right)>V\left(\theta^{\prime}, q\right)-V\left(\theta^{\prime}, q^{\prime}\right)
$$

Proof. In the case of $q^{\prime}=\mathrm{Na}$, the statement is obvious because $V(\theta, \mathrm{Na})=0, V\left(\theta^{\prime}, \mathrm{Na}\right)=0$ and $V_{\theta}(\theta, q)>0$ for all

\footnotetext{
${ }^{4}$ Note that if the utility of a type- $\theta$ SU is negative under $(q(\theta), \pi(\theta))$, his utilities under all quality-price combinations other than $(\mathrm{Na}, 0)$ are also negative according to the IC constraint in Eq. (7).
}

$q>\mathrm{Na}$. To prove the statement in the case of $q>q^{\prime}>\mathrm{Na}$, we use the Spence-Mirrlees condition or single crossing property [30]. It is easy to see that $V(\theta, q)$ in Eq. (3) satisfies the Spence-Mirrlees condition, i.e., $V_{\theta q}(\theta, q)>0$. By employing the fundamental theorem of calculus to write the difference of a function in terms of an integral of its derivative, we have:

$$
\begin{aligned}
& V(\theta, q)-V\left(\theta, q^{\prime}\right)-V\left(\theta^{\prime}, q\right)+V\left(\theta^{\prime}, q^{\prime}\right) \\
= & \int_{q^{\prime}}^{q} V_{q}(\theta, y) \mathrm{d} y-\int_{q^{\prime}}^{q} V_{q}\left(\theta^{\prime}, y\right) \mathrm{d} y \\
= & \int_{\theta^{\prime}}^{\theta}\left(\int_{q^{\prime}}^{q} V_{\theta q}(x, y) \mathrm{d} y\right) \mathrm{d} x>0
\end{aligned}
$$

The last line follows because $q>q^{\prime}, \theta>\theta^{\prime}$, and the quantity being integrated (the integrand) is positive. Q.E.D.

In what following, we will provide the necessary and sufficient conditions for a contract to be feasible, then we derive the best prices for a feasible contract with fixed quality assignment, and finally we derive the best quality assignment for the optimal contract.

\section{A. Feasibility of Contract}

We present the first necessary condition for a contract to be feasible in the following lemma.

Lemma 1: For any feasible contract $\mathscr{C}=\left\{\left(q_{t}, \pi_{t}\right)\right\}$, the following condition holds: $q_{i}>q_{j}$ if and only if $\pi_{i}>\pi_{j}$.

Proof. We prove this lemma using the IC constraint in (7). $\rightarrow$ : In this direction we will prove: if $q_{i}>q_{j}$, then $\pi_{i}>\pi_{j}$. For type- $\theta_{j}$ SUs, the following IC constraint must be satisfied:

$$
V\left(\theta_{j}, q_{j}\right)-V\left(\theta_{j}, q_{i}\right) \geq \pi_{j}-\pi_{i}
$$

from which we can find that if $q_{i}>q_{j}$, then $\pi_{j}-\pi_{i} \leq$ $V\left(\theta_{j}, q_{j}\right)-V\left(\theta_{j}, q_{i}\right)<0$, i.e., $\pi_{i}>\pi_{j}$.

$\leftarrow$ : Now we prove: if $\pi_{i}>\pi_{j}$, then $q_{i}>q_{j}$. Similarly, for type $-\theta_{i}$ SUs, the following IC constraint must be satisfied:

$$
V\left(\theta_{i}, q_{i}\right)-V\left(\theta_{i}, q_{j}\right) \geq \pi_{i}-\pi_{j}
$$

from which we can find that if $\pi_{i}>\pi_{j}$, then $V\left(\theta_{i}, q_{i}\right)-$ $V\left(\theta_{i}, q_{j}\right)>0$, which implies $q_{i}>q_{j}$ since $V(\theta, q)$ is a strictly monotone increasing function on $q$. Q.E.D.

The above lemma provides an important property for a feasible contract: a higher quality must correspond to a higher price. From Lemma 1, we can also find that if there exist qualities with the same value, the corresponding prices are also the same, and vice versa. Formally, we show this property as the following corollary.

Corollary: For any feasible contract $\mathscr{C}=\left\{\left(q_{t}, \pi_{t}\right)\right\}$, the following condition holds: $q_{i}=q_{j}$ if and only if $\pi_{i}=\pi_{j}$.

The second necessary condition for a contract to be feasible is shown in Lemma 2.

Lemma 2: For any feasible contract $\mathscr{C}=\left\{\left(q_{t}, \pi_{t}\right)\right\}$, the following condition holds: if $\theta_{i}>\theta_{j}$, then $q_{i} \geq q_{j}$.

Proof. We prove the lemma by contradiction. Assume to the contrary that there exist $\theta_{i}>\theta_{j}$ and $q_{i}<q_{j}$. Through simple transforming on the IP property in Eq. (12), we have:

$$
V\left(\theta_{i}, q_{j}\right)+V\left(\theta_{j}, q_{i}\right)>V\left(\theta_{j}, q_{j}\right)+V\left(\theta_{i}, q_{i}\right)
$$


Additionally, a feasible contract must satisfy the IC constraints for both consumer types $\theta_{i}$ and $\theta_{j}$, i.e.,

$$
\left\{\begin{aligned}
V\left(\theta_{i}, q_{i}\right)-\pi_{i} \geq V\left(\theta_{i}, q_{j}\right)-\pi_{j} & \text { for type- } \theta_{i} \text { SUs } \\
V\left(\theta_{j}, q_{j}\right)-\pi_{j} \geq V\left(\theta_{j}, q_{i}\right)-\pi_{i} & \text { for type- } \theta_{j} \text { SUs }
\end{aligned}\right.
$$

Combining the above two equations, we have:

$$
V\left(\theta_{i}, q_{i}\right)+V\left(\theta_{j}, q_{j}\right) \geq V\left(\theta_{i}, q_{j}\right)+V\left(\theta_{j}, q_{i}\right)
$$

which violates the IP property. Q.E.D.

Lemma 2 provides another important property for a feasible contract: the quality assigned to a higher type SU must be greater than that to a lower one. Reviewing the definition of consumer type, i.e., $\frac{L_{i}}{I_{i}+J_{i}+\sigma^{2}}$, the SUs close to the PO or PUs will suffer from a large interference from the PO or PUs (i.e., large $I_{i}$ or $J_{i}$ ), and accordingly lead to a small type. According to the symmetry property in radio propagation, the transmission of these SUs will also cause large interference in the PO or PUs. In a feasible contract, these SUs will be assigned to low qualities, that is, they will be allowed to transmit in low power. Thus the feasible contract can naturally reduce the interference between the primary network and secondary network.

From the above two lemmas, we can find that a feasible contract has the following structure (or necessary conditions):

$$
q_{1} \leq q_{2} \leq \ldots \leq q_{T}, \text { and } \pi_{1} \leq \pi_{2} \leq \ldots \leq \pi_{T}
$$

with $q_{i}=q_{i+1}$ if and only if $\pi_{i}=\pi_{i+1}$.

The above lemmas present the necessary conditions for a feasible contract. The sufficient conditions for a contract to be feasible are shown in the following lemma.

Lemma 3: For any contract $\mathscr{C}=\left\{\left(q_{t}, \pi_{t}\right)\right\}$, it is feasible if the following conditions hold:

- $\mathrm{Na} \leq q_{1} \leq q_{2} \leq \ldots \leq q_{T} \leq P_{\max }$

- $0 \leq \pi_{1} \leq V\left(\theta_{1}, q_{1}\right)$, and

- for all $k=2,3, \ldots, T$,

$$
\pi_{k-1}+A \leq \pi_{k} \leq \pi_{k-1}+B
$$

where $A=V\left(\theta_{k-1}, q_{k}\right)-V\left(\theta_{k-1}, q_{k-1}\right)$ and $B=$ $V\left(\theta_{k}, q_{k}\right)-V\left(\theta_{k}, q_{k-1}\right)$.

Proof. We prove the lemma by mathematical induction. We denote $\mathscr{C}(n)$ as a subset of $\mathscr{C}$ which contains the first $n$ quality-price combinations in $\mathscr{C}$, i.e., $\mathscr{C}(n)=\left\{\left(q_{t}, \pi_{t}\right) \mid t=\right.$ $1, \ldots, n\}$. Let $\mathscr{C}(n)$ be a contract for the network which contains the first $n$ consumer types of the original network.

We first verify that $\mathscr{C}(1)$ is feasible. Since there is only one consumer type, the condition for a contract to be feasible is only the IR constraint in Eq. (8). Obviously $V\left(\theta_{1}, q_{1}\right)-\pi_{1} \geq$ $0=V\left(\theta_{1}, \mathrm{Na}\right)-\pi(\mathrm{Na})$, thus $\mathscr{C}(1)$ is a feasible contract.

We then show that if $\mathscr{C}(k)$ is a feasible contract, $\mathscr{C}(k+1)$ is also feasible. To achieve this, we need to prove that (I) for the new type $\theta_{k+1}$, the IC and IR constraints are satisfied, i.e.,

$$
\left\{\begin{array}{l}
V\left(\theta_{k+1}, q_{k+1}\right)-\pi_{k+1} \geq V\left(\theta_{k+1}, q_{i}\right)-\pi_{i}, \forall i=1, \ldots, k \\
V\left(\theta_{k+1}, q_{k+1}\right)-\pi_{k+1} \geq V\left(\theta_{k+1}, \mathrm{Na}\right)-\pi(\mathrm{Na})=0
\end{array}\right.
$$

and (II) for the existing types $\theta_{1}, \ldots, \theta_{k}$, the IC constraints are still satisfied in the presence of type $\theta_{k+1}$, i.e.,

$$
V\left(\theta_{i}, q_{i}\right)-\pi_{i} \geq V\left(\theta_{i}, q_{k+1}\right)-\pi_{k+1}, \forall i=1, \ldots, k
$$

(Proof of I) Now we prove Eq. (15), i.e., the IC and IR constraints for the new type $\theta_{k+1}$. Since $\mathscr{C}(k)$ is feasible, the IC constraint for type $\theta_{k}$ must be satisfied, i.e.,

$$
V\left(\theta_{k}, q_{i}\right)-\pi_{i} \leq V\left(\theta_{k}, q_{k}\right)-\pi_{k}, \forall i=1, \ldots, k
$$

Besides, using the right inequality in Eq. (14), we have:

$$
\pi_{k+1} \leq \pi_{k}+V\left(\theta_{k+1}, q_{k+1}\right)-V\left(\theta_{k+1}, q_{k}\right)
$$

Combining the above two equations, we have:

$V\left(\theta_{k}, q_{i}\right)-\pi_{i}+\pi_{k+1} \leq V\left(\theta_{k}, q_{k}\right)+V\left(\theta_{k+1}, q_{k+1}\right)-V\left(\theta_{k+1}, q_{k}\right)$

for all $i=1, \ldots, k$. Through simple transforming, we can write the above equation as follows:

$V\left(\theta_{k+1}, q_{k+1}\right)-\pi_{k+1} \geq V\left(\theta_{k}, q_{i}\right)-V\left(\theta_{k}, q_{k}\right)+V\left(\theta_{k+1}, q_{k}\right)-\pi_{i}$

for all $i=1, \ldots, k$. Note that $\theta_{k+1}>\theta_{k}$ and $q_{k} \geq q_{i}, \forall i=$ $1, \ldots, k$, and using IP property, we have:

$$
V\left(\theta_{k+1}, q_{k}\right)-V\left(\theta_{k+1}, q_{i}\right) \geq V\left(\theta_{k}, q_{k}\right)-V\left(\theta_{k}, q_{i}\right)
$$

Substituting the above equation into Eq. (17), we can prove the IC constraint for the new type $\theta_{k+1}$, i.e.,

$$
V\left(\theta_{k+1}, q_{k+1}\right)-\pi_{k+1} \geq V\left(\theta_{k+1}, q_{i}\right)-\pi_{i}, \forall i=1, \ldots, k
$$

Further, we can easily prove the IR constraint for type $\theta_{k+1}$, as we notice that $V\left(\theta_{k+1}, q_{i}\right)-\pi_{i} \geq V\left(\theta_{i}, q_{i}\right)-\pi_{i} \geq 0$, $\forall i \leq k$, since the IR constraints for all type- $\theta_{i}$ are satisfied.

(Proof of II) Here we prove Eq. (16), i.e., the IC constraints for the existing types $\theta_{i}, \forall i=1, \ldots, k$, in the presence of type $\theta_{k+1}$. Using the left inequality in Eq. (14), we have:

$$
\pi_{k}+V\left(\theta_{k}, q_{k+1}\right)-V\left(\theta_{k}, q_{k}\right) \leq \pi_{k+1}
$$

Besides, since $\mathscr{C}(k)$ is feasible, the IC constraints for type $\theta_{i}$, $\forall i=1, \ldots, k$, must be satisfied, i.e.,

$$
V\left(\theta_{i}, q_{k}\right)-\pi_{k} \leq V\left(\theta_{i}, q_{i}\right)-\pi_{i}, \forall i=1, \ldots, k
$$

Combining the above two equations, we have:

$V\left(\theta_{i}, q_{k}\right)+V\left(\theta_{k}, q_{k+1}\right)-V\left(\theta_{k}, q_{k}\right) \leq V\left(\theta_{i}, q_{i}\right)-\pi_{i}+\pi_{k+1}$ for all $i=1, \ldots, k$. Through simple transforming, we can write the above equation as follows:

$V\left(\theta_{i}, q_{i}\right)-\pi_{i} \geq V\left(\theta_{i}, q_{k}\right)+V\left(\theta_{k}, q_{k+1}\right)-V\left(\theta_{k}, q_{k}\right)-\pi_{k+1}$

for all $i=1, \ldots, k$. Note that $\theta_{k} \geq \theta_{i}$ and $q_{k+1} \geq q_{k}, \forall i=$ $1, \ldots, k$, and using IP property, we have:

$$
V\left(\theta_{k}, q_{k+1}\right)-V\left(\theta_{k}, q_{k}\right) \geq V\left(\theta_{i}, q_{k+1}\right)-V\left(\theta_{i}, q_{k}\right)
$$

Substituting the above equation into Eq. (18), we can prove the IC constraints for the existing type $\theta_{i}, \forall i=1, \ldots, k$, i.e.,

$$
V\left(\theta_{i}, q_{i}\right)-\pi_{i} \geq V\left(\theta_{i}, q_{k+1}\right)-\pi_{k+1}, \forall i=1, \ldots, k
$$

Up to present, we have proved that (i) $\mathscr{C}(1)$ is feasible, and (ii) if $\mathscr{C}(k)$ is feasible, then $\mathscr{C}(k+1)$ is feasible. It follows that the $\mathscr{C}=\mathscr{C}(T)$ is feasible. Q.E.D.

It is easy to see that the sufficient conditions in Lemma 3 are also the necessary conditions for a feasible contract. Specifically, the necessity of the first condition in Lemma 3 can be proved by Lemma 2, and the necessity of the last 


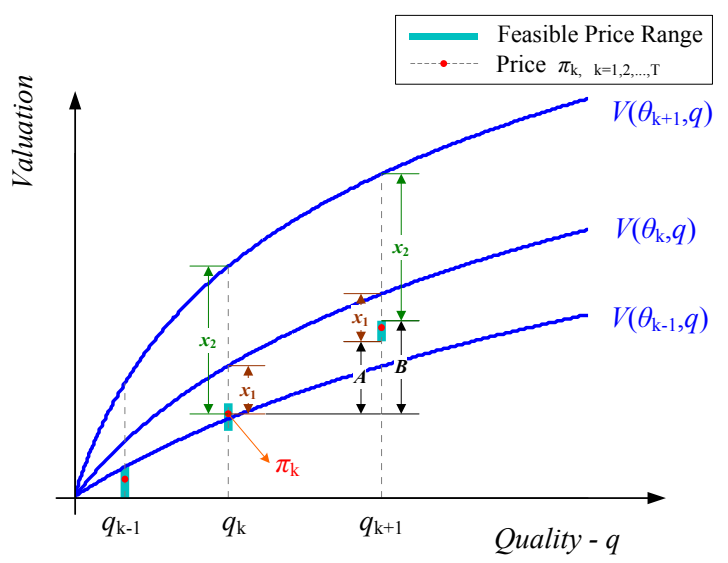

Fig. 3. An illustration of the feasible price range characterized by Lemma 3.

two conditions in Lemma 3 can be straightforwardly proved by the IR and IC constraints. In details, according to the IR constraint for type $\theta_{1}$, i.e., $V\left(\theta_{1}, q_{1}\right)-\pi_{1} \geq 0$, we can prove the second condition in Lemma 3 . According to the IC constraints for types $\theta_{k-1}$ and $\theta_{k}$, i.e., $V\left(\theta_{k-1}, q_{k-1}\right)-\pi_{k-1} \geq$ $V\left(\theta_{k-1}, q_{k}\right)-\pi_{k}$ and $V\left(\theta_{k}, q_{k}\right)-\pi_{k} \geq V\left(\theta_{k}, q_{k-1}\right)-\pi_{k-1}$, we can prove the third condition in Lemma 3.

We can also see that the conditions in Lemma 3 satisfies the necessary conditions in Eq. (13). Specifically, it is obvious that $\pi_{k} \geq \pi_{k-1}$ because $A \geq 0$ since $q_{k} \geq q_{k-1}$. Further, if $q_{k}=q_{k-1}$, then $\pi_{k-1} \leq \pi_{k} \leq \pi_{k-1}$ since $A=B=$ 0 , which implies that $\pi_{k}=\pi_{k-1}$; and if $\pi_{k}=\pi_{k-1}$, then $A \leq 0$ and $0 \leq B$, which implies that $q_{k} \leq q_{k-1}$ and $q_{k} \geq$ $q_{k-1}$, it follows that $q_{k}=q_{k-1}$. In fact, the conditions in Lemma 3 provide stricter and tighter necessary conditions for the feasible contract.

Figure 3 presents an illustration of the feasible price range for type $\theta_{k+1}$ characterized by Lemma 3 . On one hand, to make the type $\theta_{k}$ prefer quality $q_{k}$ to $q_{k+1}$, the utility of type $\theta_{k}$ on quality $q_{k+1}$ must be smaller than that on quality $q_{k}$, i.e., $V\left(\theta_{k}, q_{k+1}\right)-\pi_{k+1} \leq x_{1}=V\left(\theta_{k}, q_{k}\right)-\pi_{k}$, which implies that $\pi_{k+1} \geq \pi_{k}+A$ where $A=V\left(\theta_{k}, q_{k+1}\right)-V\left(\theta_{k}, q_{k}\right)$. On the other hand, to make the type $\theta_{k+1}$ prefer $q_{k+1}$ to $q_{k}$, the utility of type $\theta_{k+1}$ on quality $q_{k+1}$ must be greater than that on quality $q_{k}$, i.e., $V\left(\theta_{k+1}, q_{k+1}\right)-\pi_{k+1} \geq x_{2}=$ $V\left(\theta_{k+1}, q_{k}\right)-\pi_{k}$, which implies that $\pi_{k+1} \leq \pi_{k}+B$ where $B=V\left(\theta_{k+1}, q_{k+1}\right)-V\left(\theta_{k+1}, q_{k}\right)$.

\section{B. Optimality of Contract}

To derive the optimal contract, we first derive the best prices in a fixed feasible quality assignment, then we derive the best quality assignment for the optimal contract. For simplicity, we refer to a quality assignment $\left\{q_{t}\right\}$ as a feasible quality assignment, if it satisfies the constraints $q_{1} \leq q_{2} \leq \ldots \leq q_{T}$. We denote $R_{\left\{q_{t}\right\}}^{*}$ as the maximum utility the $\mathrm{PO}$ can achieve in a given feasible quality assignment $\left\{q_{t}\right\}$. Obviously, $R_{\left\{q_{t}\right\}}^{*}$ can be obtained as follows:

$$
R_{\left\{q_{t}\right\}}^{*}=\max _{\left\{\pi_{t}\right\}} \sum_{t=1}^{T} N_{t}\left(\pi_{t}-C\left(q_{t}\right)\right)
$$

subject to the price constraints in Lemma 3.

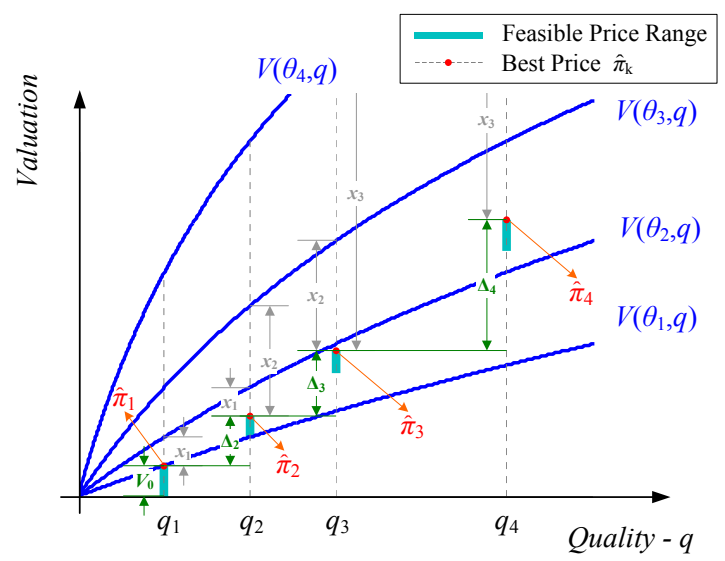

Fig. 4. An illustration of the best price assignment characterized by Lemma 4.

Lemma 4: Let $\mathscr{C}=\left\{\left(q_{t}, \pi_{t}\right)\right\}$ be a feasible contract with fixed qualities $q_{1} \leq q_{2} \leq \ldots \leq q_{T}$. Then the unique best price assignment for Eq. (19), denoted by $\left\{\hat{\pi}_{t}\right\}$, is given by:

$$
\hat{\pi}_{k}=\hat{\pi}_{k-1}+V\left(\theta_{k}, q_{k}\right)-V\left(\theta_{k}, q_{k-1}\right)
$$

for all $k=2,3, \ldots, T$, and $\hat{\pi}_{1}=V\left(\theta_{1}, q_{1}\right)$.

Proof. Obviously the prices in Eq. (20) form a feasible price assignment, that is, they satisfy the conditions in Lemma 3.

We first show that the prices given by Eq. (20) maximize the utility of the PO. Note that, in the case of fixed quality assignment, we can achieve the maximum utility through maximizing the sum of prices, i.e., $\sum_{t=1}^{T} N_{t} \pi_{t}$, since the sum of cost, i.e., $\sum_{t=1}^{T} N_{t} C\left(q_{t}\right)$, is constant. Assume that there is a price assignment $\left\{\tilde{\pi}_{t}\right\}$ such that $\sum_{t=1}^{T} N_{t} \tilde{\pi}_{t}>\sum_{t=1}^{T} N_{t} \hat{\pi}_{t}$. It is easy to see that there is at least one price $\tilde{\pi}_{k}>\hat{\pi}_{k}$. To make the contract be feasible, the following constraint on $\left\{\tilde{\pi}_{t}\right\}$ must be satisfied according to Lemma 3:

$$
\tilde{\pi}_{k} \leq \tilde{\pi}_{k-1}+V\left(\theta_{k}, q_{k}\right)-V\left(\theta_{k}, q_{k-1}\right)
$$

Combining the above equation with Eq. (20), we have:

$$
\tilde{\pi}_{k-1}>\hat{\pi}_{k}-V\left(\theta_{k}, q_{k}\right)+V\left(\theta_{k}, q_{k-1}\right)=\hat{\pi}_{k-1}
$$

Continuing the above process, we can finally obtain that $\tilde{\pi}_{1}>$ $\hat{\pi}_{1}=V\left(\theta_{1}, q_{1}\right)$, which violates the IR constraint for type $\theta_{1}$. Therefore, there does not exist any feasible price assignment $\left\{\tilde{\pi}_{t}\right\}$ such that $\sum_{t=1}^{T} N_{t} \tilde{\pi}_{t}>\sum_{t=1}^{T} N_{t} \hat{\pi}_{t}$, which implies that the utility is maximized under the price assignment $\left\{\hat{\pi}_{t}\right\}$.

Then we show that $\left\{\hat{\pi}_{t}\right\}$ is the unique best price assignment. Assume to the contract that there exists a feasible price assignment $\left\{\tilde{\pi}_{t}\right\} \neq\left\{\hat{\pi}_{t}\right\}$ such that $\sum_{t=1}^{T} N_{t} \tilde{\pi}_{t}=\sum_{t=1}^{T} N_{t} \hat{\pi}_{t}$. Obviously there is at least one price $\tilde{\pi}_{k} \neq \hat{\pi}_{k}$. Without loss of generality, we assume that $\tilde{\pi}_{k}<\hat{\pi}_{k}$. It is easy to see that there must exist another price $\tilde{\pi}_{j}>\hat{\pi}_{j}$. Using the same method, we can obtain that $\tilde{\pi}_{1}>\hat{\pi}_{1}=V\left(\theta_{1}, q_{1}\right)$. Therefore, there does not exist any feasible price assignment $\left\{\tilde{\pi}_{t}\right\} \neq\left\{\hat{\pi}_{t}\right\}$ such that $\sum_{t=1}^{T} N_{t} \tilde{\pi}_{t}=\sum_{t=1}^{T} N_{t} \hat{\pi}_{t}$, which implies that the best price assignment $\left\{\hat{\pi}_{t}\right\}$ given by Eq. (20) is unique. Q.E.D.

Using the notations $\Delta_{1}=0$ and $\Delta_{k}=V\left(\theta_{k}, q_{k}\right)-$ $V\left(\theta_{k}, q_{k-1}\right), \forall k=2, \ldots, T$, we can simply write the best 
prices provided by Lemma 4 as follows:

$$
\hat{\pi}_{k}=V\left(\theta_{1}, q_{1}\right)+\sum_{i=1}^{k} \Delta_{i}, \quad \forall k=1, \ldots, T
$$

Figure 4 presents an illustration of the best price assignment characterized by Lemma 4 . Note that we cut off the valuation curve of type $\theta_{4}$ due to space limitations. We can find from the figure that $\hat{\pi}_{1}=V_{0}=V\left(\theta_{1}, q_{1}\right), \hat{\pi}_{2}=\hat{\pi}_{1}+\Delta_{2}=V\left(\theta_{1}, q_{1}\right)+$ $\Delta_{2}, \hat{\pi}_{3}=\hat{\pi}_{2}+\Delta_{3}=V\left(\theta_{1}, q_{1}\right)+\Delta_{2}+\Delta_{3}$, and so on.

Lemma 4 suggests that, for any fixed feasible qualities, the best price assignment given by Eq. (20) or (21) is unique. Therefore, the maximum utility of the PO, denoted by $R^{*}$, can be carried out with the converse induction, i.e.,

$$
R^{*}=\max _{\left\{q_{t}\right\}} R_{\left\{q_{t}\right\}}^{*}
$$

subject to $q_{1} \leq q_{2} \leq \ldots \leq q_{T}$. Note that the constraints $q_{1} \leq q_{2} \leq \ldots \leq q_{T}$ are necessary to ensure the feasibility of the contract.

Substituting Eq. (21) for the prices in Eq. (19), we have:

$$
R_{\left\{q_{t}\right\}}^{*}=\sum_{t=1}^{T} N_{t}\left(V\left(\theta_{1}, q_{1}\right)+\sum_{i=1}^{t} \Delta_{i}-C\left(q_{t}\right)\right)
$$

Re-arranging the above equation by putting the terms related to the same quality variable together, we can equivalently write $R_{\left\{q_{t}\right\}}^{*}$ as follows:

$$
R_{\left\{q_{t}\right\}}^{*}=\sum_{t=1}^{T}\left(N_{t} V\left(\theta_{t}, q_{t}\right)-N_{t} C\left(q_{t}\right)+\Lambda_{t} \sum_{i=t+1}^{T} N_{i}\right)
$$

where $\Lambda_{k}=V\left(\theta_{k}, q_{k}\right)-V\left(\theta_{k+1}, q_{k}\right), \forall k<T$, and $\Lambda_{T}=0$.

For simplicity, we use the notation $G_{t}=N_{t} V\left(\theta_{t}, q_{t}\right)-$ $N_{t} C\left(q_{t}\right)+\Lambda_{t} \sum_{i=t+1}^{T} N_{i}$, thus we can write Eq. (24) as $R_{\left\{q_{t}\right\}}^{*}=\sum_{t=1}^{T} G_{t}$. It is worth noting that $G_{t}$ is only related to $q_{t}$, i.e., the quality assigned to type $\theta_{t}$, and independent of the qualities assigned to other types. Therefore, the best quality assignment for Eq. (22), denoted by $\left\{\hat{q}_{t}\right\}$, can be computed by separately maximizing each of $G_{t}, \forall t=1,2, \ldots, T$.

We denote $\tilde{q}_{t}$ as the quality which maximizes $G_{t}$, i.e.,

$$
\tilde{q}_{t}=\arg \max _{q_{t}} G_{t}
$$

for all $t=1,2, \ldots, T$. Obviously, $\tilde{q}_{t}$ can be found at the boundary points ( $\mathrm{Na}$ and $P_{\max }$ ) or at the critical points, i.e., the qualities satisfying $\frac{\mathrm{d} G_{t}}{\mathrm{~d} q_{t}}=0$ and $\frac{\mathrm{d}^{2} G_{t}}{\mathrm{~d} q_{t}^{2}} \leq 0$, according to Fermat's theorem for stationary points. Figure 5 presents an illustration of the best quality for $G_{t}$, where $G_{t}^{\prime}$ denotes the first derivative of $G_{t}$, i.e., $\frac{\mathrm{d} G_{t}}{\mathrm{~d} q_{t}}$. From Figure 5 , we can find that, $\tilde{q}_{2}$, the best quality for $G_{2}$, is at its critical point $p_{2}$, while $\tilde{q}_{3}$ is at the right boundary point $P_{\max }$ since the critical point for $G_{3}$ goes beyond the available quality range. Besides, $\tilde{q}_{1}$ is at the left boundary point $\mathrm{Na}$, since $G_{1}(\mathrm{Na})=0$ and $G_{1}\left(q_{1}\right)<0$ for all $q_{1} \in\left[P_{\min }, P_{\max }\right]$.

It is obvious that $\left\{\tilde{q}_{t}\right\}$ is exactly the solution for Eq. (22), i.e., $\left\{\hat{q}_{t}\right\}=\left\{\tilde{q}_{t}\right\}$, if it is a feasible quality assignment. Thus we have the following proposition.

Proposition 2: With uniformly distribution of consumer types, i.e., $N_{1}=N_{2}=\ldots=N_{T}$ and $\theta_{2}-\theta_{1}=\theta_{3}-\theta_{2}=$

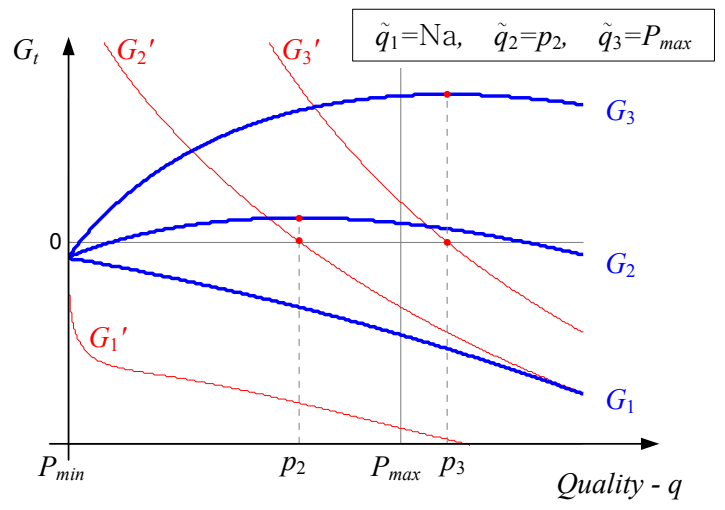

Fig. 5. An illustration of the best quality for $G_{t}$.

$\ldots=\theta_{T}-\theta_{T-1}$, the best quality assignment $\left\{\hat{q}_{t}\right\}=\left\{\tilde{q}_{t}\right\}$ where $\tilde{q}_{t}=\arg \max _{q_{t}} G_{t}$.

The proposition can be easily proved as we notice that, in the case of uniformly distribution of consumer types, $\left\{\tilde{q}_{t}\right\}$ is exactly in the increasing order. However, in the case of general distribution of consumer types, $\left\{\tilde{q}_{t}\right\}$ may not be in the increasing order, that is, it may be infeasible. For example, for a type $\theta_{k}$ with few consumers, i.e., small $N_{k}$, the value of $G_{k}$ is greatly determined by $\Lambda_{k}$, thus the best quality for $G_{k}$ will tend to be small since $\Lambda_{k}$ is decreasing with the quality.

We denote a sub-sequence of $\left\{q_{t}\right\}$, say $\left\{q_{i}, q_{i+1}, \ldots, q_{j}\right\}$, as an infeasible sub-sequence, if $q_{i} \geq q_{i+1} \geq \ldots \geq q_{j}$ and $q_{i}>q_{j}$. For example, in a quality assignment $\left\{q_{t}\right\}=$ $\{1,3,3,2,6,5\}$, there are two infeasible sub-sequences, i.e., $\left\{q_{2}, q_{3}, q_{4}\right\}$ and $\left\{q_{5}, q_{6}\right\}$. Obviously, for any infeasible quality assignment $\left\{q_{t}\right\}$, there is at least one infeasible sub-sequence. It is necessary to design a mechanism to deal with the infeasible sub-sequences of $\left\{\tilde{q}_{t}\right\}$. The following proposition is essential for designing such a mechanism.

Proposition 3: Let $X_{1}(x)$ and $X_{2}(x)$ be concave functions on $x$. If $\tilde{x}_{1} \geq \tilde{x}_{2}$ where $\tilde{x}_{1}=\arg \max _{x_{1}} X_{1}\left(x_{1}\right)$ and $\tilde{x}_{2}=$ $\arg \max _{x_{2}} X_{2}\left(x_{2}\right)$, then $\hat{x}_{1}=\hat{x}_{2}$ where

$$
\left\{\hat{x}_{1}, \hat{x}_{2}\right\}=\arg \max _{x_{1}, x_{2}} \sum_{i=1}^{2} X_{i}\left(x_{i}\right), \text { s.t. } x_{1} \leq x_{2}
$$

Proof. The statement is obvious if $\tilde{x}_{1}=\tilde{x}_{2}$, thus we focus on the case of $\tilde{x}_{1}>\tilde{x}_{2}$, as shown in Figure 6 .

We can prove the statement if we show that, for arbitrary $x_{1}<x_{2}$, we can find an $x^{\prime}$ such that $\sum_{i=1}^{2} X_{i}\left(x^{\prime}\right)>$ $\sum_{i=1}^{2} X_{i}\left(x_{i}\right)$. There are two cases. If $x_{1}<\tilde{x}_{2}$, e.g., $x_{1}=a$, the circle in Figure 6, we have $\sum_{i=1}^{2} X_{i}\left(x_{i}\right)<\sum_{i=1}^{2} X_{i}\left(\tilde{x}_{2}\right)$, i.e., $x^{\prime}=\tilde{x}_{2}$, since $X_{1}\left(x_{1}\right)<X_{1}\left(\tilde{x}_{2}\right)$ and $X_{2}\left(x_{2}\right) \leq X_{2}\left(\tilde{x}_{2}\right)$. If $x_{1} \geq \tilde{x}_{2}$, e.g., $x_{1}=b$, the square in Figure 6 , we have $\sum_{i=1}^{2} X_{i}\left(x_{i}\right)<\sum_{i=1}^{2} X_{i}\left(x_{1}\right)$, i.e., $x^{\prime}=x_{1}$, since $X_{2}\left(x_{2}\right)<X_{2}\left(x_{1}\right)$ for all $x_{2}>x_{1} \geq \tilde{x}_{2}$. Q.E.D.

Proposition 3 can be extended to a more general form: if $\tilde{x}_{1} \geq \tilde{x}_{2} \geq \ldots \geq \tilde{x}_{K}$ where $\tilde{x}_{i}=\arg \max _{x_{i}} X_{i}\left(x_{i}\right)$, then $\hat{x}_{1}=$ $\hat{x}_{2}=\ldots=\hat{x}_{K}$ where $\left\{\hat{x}_{k}\right\}=\arg \max _{\left\{x_{k}\right\}} \sum_{k=1}^{K} X_{k}\left(x_{k}\right)$ subject to $x_{1} \leq x_{2} \leq \ldots \leq x_{K}$. Further, the optimal solution $\hat{x}_{i}$ can be easily obtained by maximizing $\sum_{k=1}^{K} X_{k}(x)$, i.e., $\hat{x}_{i}=\arg \max _{x} \sum_{k=1}^{K} X_{k}(x)$.

By means of Proposition 3, we design a dynamic algorithm to make the infeasible sub-sequences in any quality assignment 


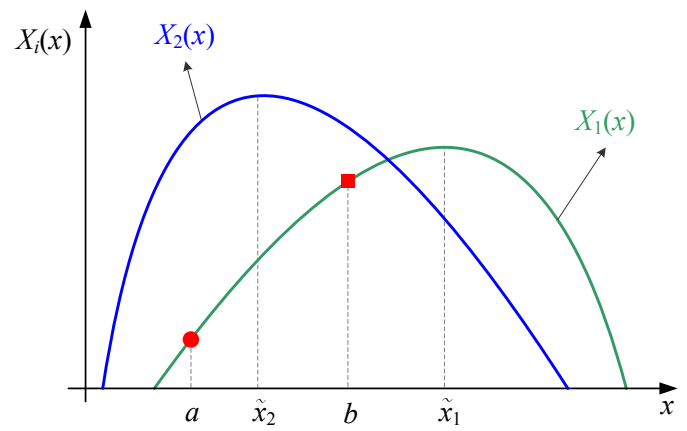

Fig. 6. An illustration of $X_{1}(x)$ and $X_{2}(x)$ in Proposition 3.

to be feasible. The algorithm starts with $\left\{\tilde{q}_{t}\right\}$ and iteratively replaces the infeasible sub-sequence of $\left\{\tilde{q}_{t}\right\}$ by any feasible sub-sequence. The detail algorithm is shown as follows: ${ }^{5}$

1) initiate $\hat{q}_{t}=\tilde{q}_{t}=\arg \max _{q_{t}} G_{t}, \forall t=1,2, \ldots, T$

2) while $\left\{\hat{q}_{t}\right\}$ is not feasible, do:

- find an infeasible sub-sequence $\left\{\hat{q}_{i}, \hat{q}_{i+1}, \ldots, \hat{q}_{j}\right\}$

- set $\hat{q}_{k}=\arg \max _{q} \sum_{t=i}^{j} G_{t}(q), \forall k=i, i+1, \ldots, j$

To provide the computational complexity of the above dynamic algorithm, we use notations $\mu_{t}=\hat{q}_{t+1}-\hat{q}_{t}, t=$ $1,2, \ldots, T-1$. Obviously that (i) if $\mu_{i} \leq 0, \mu_{i+1} \leq 0, \ldots, \mu_{j} \leq$ 0 with at least one strict inequality, then $\left\{\hat{q}_{i}, \hat{q}_{i+1}, \ldots, \hat{q}_{j+1}\right\}$ is an infeasible sub-sequence, and (ii) $\left\{\hat{q}_{t}\right\}$ is feasible if and only if $\mu_{t} \geq 0, \forall t=1,2, \ldots, T-1$. We can find that in each iteration of the algorithm, at least one negative $\mu_{t}$ is set to be zero. Note that the zero $\mu_{t}$ will hold at zero in the future iteration. Thus the maximum iteration number is $T-1$. Further, the main computational burden in each iteration is to solve the singlevariable optimization problem $\hat{q}_{k}=\arg \max _{q} \sum_{t=i}^{j} G_{t}(q)$, which can be easily achieved by binary searching.

Figure 7 illustrates the dynamic algorithm in a network with 6 consumer types. Sub-figure (a) is the quality assignment at the initiating stage, i.e., $\hat{q}_{t}=\tilde{q}_{t}=\arg \max _{q_{t}} G_{t}, \forall t=$ $1,2, \ldots, 6$. Obviously there are two infeasible sub-sequences $\left\{\hat{q}_{1}, \hat{q}_{2}\right\}$ and $\left\{\hat{q}_{4}, \hat{q}_{5}\right\}$. Sub-figure (b) presents the updated quality assignment by replacing the infeasible sub-sequence $\left\{\hat{q}_{1}, \hat{q}_{2}\right\}$ with $q^{\prime}=\arg \max _{q} \sum_{t=1}^{2} G_{t}(q)$. Similarly, sub figure-(c) presents the updated quality assignment by replacing $\left\{\hat{q}_{4}, \hat{q}_{5}\right\}$ with $q^{\prime}=\arg \max _{q} \sum_{t=4}^{5} G_{t}(q)$. Note that there is a new infeasible sub-sequence $\left\{\hat{q}_{3}, \hat{q}_{4}, \hat{q}_{5}\right\}$ after the stage in (c). Finally, sub-figure (d) present the best quality assignment by replacing $\left\{\hat{q}_{3}, \hat{q}_{4}, \hat{q}_{5}\right\}$ with $q^{\prime}=\arg \max _{q} \sum_{t=3}^{5} G_{t}(q)$. We can see that $\mu_{1}$ is set to be zero in the process of Sub-figure (b), $\mu_{4}$ is set to be zero in the process of Sub-figure (c), and $\mu_{3}$ is set to zero in the process of Sub-figure (d).

Based on the above lemmas and propositions, we have the following theorem:

Theorem 1: Contract $\mathscr{C}=\left\{\left(\hat{q}_{t}, \hat{\pi}_{t}\right)\right\}$ is optimal, if $\left\{\hat{q}_{t}\right\}$ is the outcome of the above dynamic algorithm and $\left\{\hat{\pi}_{t}\right\}$ is given by Lemma 4.

\footnotetext{
${ }^{5}$ Note that $G_{t}$ is concave in the case of large $q_{t}$, since $\partial^{2} \Lambda_{t} / \partial q_{t}^{2}$ tends to be zero if $q_{t}$ is large, and $\partial^{2}\left(V\left(\theta_{t}, q_{t}\right)-C\left(q_{t}\right)\right) / \partial q_{t}^{2}=S_{q q}\left(\theta_{t}, q_{t}\right)$ which is always small than zero.
}

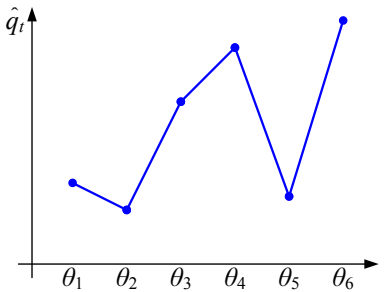

(a)

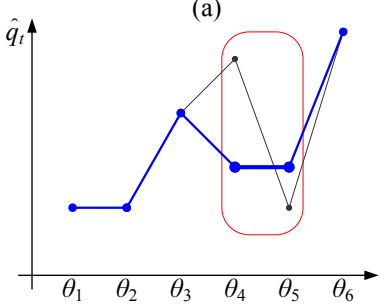

(c)

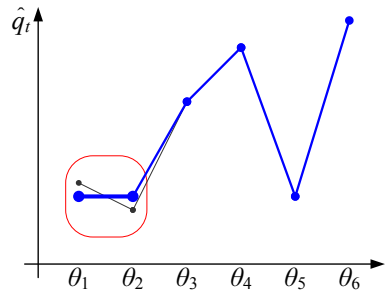

(b)

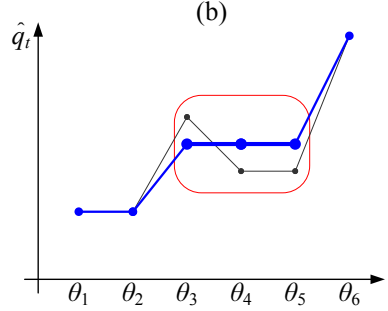

(d)
Fig. 7. Illustrating the process of the dynamic algorithm.

\section{Practical Implementation Issues}

In above discussion, we assume the spectrum for sale is unlimited, that is, the PO has a sufficient number of idle channels for sale, i.e., $M \geq \sum_{t=1}^{T} N_{t}$, where $M$ is the number of idle channels and $\sum_{t=1}^{T} N_{t}$ is the number of SUs. In a practical cognitive radio network, however, it is more realistic to assume that the spectrum for sale is limited, that is, the number of idle channels may be less than the number of SUs, i.e., $M<\sum_{t=1}^{T} N_{t}$, in particular for the network with a large number of SUs. We refer to this as limited spectrum trading. Note that in limited spectrum trading, there must be some SUs who can not acquire channels since each channel can only be sold to one SU, thus the overall utility of the PO is not given by Eq. (9) any longer.

We first analyze the utility the PO can achieve from a type$\theta_{t} \mathrm{SU}$ in an optimal contract $\mathscr{C}=\left\{\left(\hat{q}_{t}, \hat{\pi}_{t}\right)\right\}$. Substituting the quality-price for type- $\theta_{t}$, i.e., $\left(\hat{q}_{t}, \hat{\pi}_{t}\right)$, into Eq. (2), we have:

$$
R\left(\hat{q}_{t}\right)=\hat{\pi}_{t}-C\left(\hat{q}_{t}\right)
$$

where $\hat{\pi}_{t}$ is the best price assignment given by Eq. (21).

The difference between the utilities the PO achieved from a type- $\theta_{t}$ SU and a type- $\theta_{t+1}$ SU can be written as:

$$
\begin{aligned}
& R\left(\hat{q}_{t+1}\right)-R\left(\hat{q}_{t}\right)=\Delta_{t+1}-C\left(\hat{q}_{t+1}\right)+C\left(\hat{q}_{t}\right) \\
& =V\left(\theta_{t+1}, \hat{q}_{t+1}\right)-V\left(\theta_{t+1}, \hat{q}_{t}\right)-C\left(\hat{q}_{t+1}\right)+C\left(\hat{q}_{t}\right) \\
& =S\left(\theta_{t+1}, \hat{q}_{t+1}\right)-S\left(\theta_{t+1}, \hat{q}_{t}\right)
\end{aligned}
$$

The following proposition is essential for analyzing the above utility difference.

Proposition 4: For any optimal contract $\mathscr{C}=\left\{\left(\hat{q}_{t}, \hat{\pi}_{t}\right)\right\}$, the following conditions hold: $\hat{q}_{t} \leq q_{t}^{*}$, where $q_{t}^{*}=q\left(\theta_{t}\right)^{*}=$ $\arg \max _{q} S\left(\theta_{t}, q\right)$ is the social optimal quality which maximizes the social surplus $S\left(\theta_{t}, q\right)$.

Proof. It is obvious that $G_{t}=N_{t} S\left(\theta_{t}, q_{t}\right)+\Lambda_{t} \sum_{i=t+1}^{T} N_{i}$ and $\left.\frac{\partial G_{t}}{\partial q_{t}}\right|_{q_{t}=q_{t}^{*}}<0$, since $\left.\frac{\partial \Lambda_{t}}{\partial q_{t}}\right|_{q_{t}=q_{t}^{*}}<0$ and $\left.\frac{\partial S\left(\theta_{t}, q_{t}\right)}{\partial q_{t}}\right|_{q_{t}=q_{t}^{*}}=0$. Thus there must be a quality $q_{t}=q_{t}^{*}-\varepsilon$ such that $G_{t}\left(\theta_{t}, q_{t}\right)>G_{t}\left(\theta_{t}, q_{t}^{*}\right)$ where $\varepsilon$ is an arbitrary small positive number. Q.E.D.

From Proposition 4 we can see that $\hat{q}_{t} \leq \hat{q}_{t+1} \leq q_{t+1}^{*}$. Substituting above equation into Eq. (28) and noticing that 
$S\left(\theta_{t+1}, q\right)$ is increasing with $q$ when $q \leq q_{t+1}^{*}$, we can obtain $R\left(\hat{q}_{t+1}\right) \geq R\left(\hat{q}_{t}\right)$. In other words, the PO can achieve higher utility from a higher type SU than a lower one in an optimal contract. Thus in limited spectrum trading, the PO can maximize his utility by selectively selling the channels to the SUs with high type. This is just the basic idea for designing the optimal contract in limited spectrum trading.

We refer to type $\theta_{t_{0}}$ as critical type if the following conditions hold: $\sum_{t=t_{0}}^{T} N_{t} \geq M$ and $\sum_{t=t_{0}+1}^{T} N_{t}<M$. Obviously the PO will sell the channels to the SUs upon the critical type, or equivalently the $\mathrm{PO}$ will set quality $q=\mathrm{Na}$ for the SUs below critical type. Thus the optimal contract $\mathscr{C}=\left\{\left(\hat{q}_{t}, \hat{\pi}_{t}\right)\right\}$ in limited spectrum trading can be written as:

$$
\begin{cases}\left(\hat{q}_{t}, \hat{\pi}_{t}\right)=(\mathrm{Na}, 0), & \forall t<t_{0} \\ \left(\hat{q}_{t}, \hat{\pi}_{t}\right)=\left(\hat{q}_{t}^{\prime}, \hat{\pi}_{t}^{\prime}\right), & \forall t \geq t_{0}\end{cases}
$$

where $\left(\hat{q}_{t}^{\prime}, \hat{\pi}_{t}^{\prime}\right), \forall t \geq t_{0}$, are the optimal quality and price assignment for the critical network which consists of $M$ SUs with highest types in the original network. Obviously in critical network, the set of consumer type is $\Theta=$ $\left\{\theta_{t_{0}}, \theta_{t_{0}+1}, \ldots, \theta_{T}\right\}$, and the number of SUs with each type $\theta_{t}$ is $N_{t}^{\prime}$, where $N_{t_{0}}^{\prime}=M-\sum_{t=t_{0}+1}^{T} N_{t}$ and $N_{t}^{\prime}=N_{t}, \forall t>t_{0}$. The optimal quality and price assignment (i.e., optimal contract) for critical network, i.e., $\mathscr{C}^{\prime}=\left\{\left(\hat{q}_{t}^{\prime}, \hat{\pi}_{t}^{\prime}\right) \mid \forall t \geq t_{0}\right\}$, can be easily derived according to the dynamic algorithm in Section IV-B.

\section{OPTIMAL CONTRACT IN CONTINUOUS-CONSUMER-TYPE MODEL}

In this section, we analyze a more general model, the continuous-consumer-type model, in which there are a continuum of consumers. The consumer type, $\theta$, is distributed with a probability distribution $f(\theta)$ and a continuous cumulative distribution $F(\theta)$ on the interval $\Theta=\left[\theta_{s}, \theta_{e}\right]$. Similar to the discrete model, we first consider the case that the PO has a sufficient number of channels. Thus the (expected) utility of the PO can be written as:

$$
R=\int_{\theta_{s}}^{\theta_{e}}(\pi(\theta)-C(q(\theta))) f(\theta) \mathrm{d} \theta
$$

Similarly, for the contract to be feasible, it must also satisfy both IC and IR constraints for all consumer types. The optimal contract defined in (10) can be rewritten as:

$$
\{(q(\theta), \pi(\theta))\}=\arg \max _{\substack{\{q(\theta)\} \\\{\pi(\theta)\}}} \int_{\theta_{s}}^{\theta_{e}}(\pi(\theta)-C(q(\theta))) f(\theta) \mathrm{d} \theta
$$

subject to the IC and IR constraints.

The continuous model can be viewed as a convergence of the discrete model in the dense of consumer types. In fact, as we assume the consumer types in discrete model become very dense, i.e., $\theta_{i+1}-\theta_{i} \rightarrow 0$, the discrete model will converge to a continuous model.

Obviously, the necessary conditions for the feasible contract in discrete model, i.e., Lemma 1 and 2, are also adapted to the continuous model. Thus we can derive the necessary conditions in continuous model from those in discrete model. Assume that $q(\theta)$ and $\pi(\theta)$ are continuous and differentiable in $\Theta$, the necessary conditions for a contract to be feasible in the continuous model are shown in following corollaries.

Corollary 1: For any feasible contract $\mathscr{C}$, the following condition holds: $q_{\theta}(x)>0$ if and only if $\pi_{\theta}(x)>0, \forall x \in \Theta$.

Proof. Note that $q_{\theta}(x)=\lim _{\sigma \rightarrow 0} \frac{q(x+\sigma)-q(x)}{\sigma}$ and $\pi_{\theta}(x)=$ $\lim _{\sigma \rightarrow 0} \frac{\pi(x+\sigma)-\pi(x)}{\sigma}$. According to Lemma 1, we have $q(x+$ $\sigma)>q(x)$ if and only if $\pi(x+\sigma)>\pi(x)$. It follows that $q_{\theta}(x)>0$ if and only if $\pi_{\theta}(x)>0, \forall x \in \Theta$. Q.E.D.

Corollary 2: For any feasible contract $\mathscr{C}$, the following condition holds: $q_{\theta}(x) \geq 0, \forall x \in \Theta$.

Proof. The statement is obvious since $q\left(\theta_{1}\right) \geq q\left(\theta_{2}\right)$ for any $\theta_{1}>\theta_{2}$, according to Lemma 2. Q.E.D.

From Corollary 1 and Corollary 2, we can easily find that a feasible contract in continuous model has the following structure (or necessary conditions):

$$
q_{\theta}(x) \geq 0, \text { and } \pi_{\theta}(x) \geq 0, \forall x \in \Theta
$$

with $q_{\theta}(x)=0$ if and only if $\pi_{\theta}(x)=0$.

The sufficient conditions for a contract to be feasible in continuous model can also be derived from those in discrete model. We show the sufficient conditions in Corollary 3.

Corollary 3: For any contract $\mathscr{C}=\{(q(\theta), \pi(\theta))\}$, it is feasible if the following conditions hold:

- $q_{\theta}(x) \geq 0$

- $0 \leq \pi\left(\theta_{s}\right) \leq V\left(\theta_{s}, q\left(\theta_{s}\right)\right)$, and

- $\pi_{\theta}(x)=V_{q}(x, q(x)) \cdot q_{\theta}(x), \forall x \in \Theta$. ${ }^{6}$

Proof. The first two conditions can be easily derived from those in discrete model, i.e., the first two conditions in Lemma 4. Thus we will focus on the proof of the third condition.

As mentioned previously, we can view the continuous model as a discrete model with infinite dense consumer types, i.e., $\theta_{i+1}=\theta_{i}+\sigma$ where $\sigma \rightarrow 0$. Substituting the $\theta_{k}$ and $\theta_{k-1}$ in Eq. (14) with $x$ and $x-\sigma$, we can rewrite Eq. (14) as follows:

$$
\pi(x-\sigma)+A \leq \pi(x) \leq \pi(x-\sigma)+B
$$

where $A=V(x-\sigma, q(x))-V(x-\sigma, q(x-\sigma))$ and $B=$ $V(x, q(x))-V(x, q(x-\sigma))$.

From the right inequality of Eq. (33), we have:

$$
\begin{aligned}
\pi_{\theta}(x) & =\lim _{\sigma \rightarrow 0} \frac{\pi(x)-\pi(x-\sigma)}{\sigma} \\
& \leq \lim _{\sigma \rightarrow 0} \frac{V(x, q(x))-V(x, q(x-\sigma))}{\sigma} \\
& =V_{q}(x, q(x)) \cdot q_{\theta}(x)
\end{aligned}
$$

Again, from the left inequality of Eq. (33), we have:

$$
\begin{aligned}
\pi_{\theta}(x) & \geq V_{q}(x-\sigma, q(x)) \cdot q_{\theta}(x) \\
& =V_{q}(x, q(x)) \cdot q_{\theta}(x)
\end{aligned}
$$

The last line follows because $\sigma \rightarrow 0$. Combining Eqs. (34) and (35), we have $\pi_{\theta}(x)=V_{q}(x, q(x)) \cdot q_{\theta}(x), \forall x \in \Theta$. Q.E.D.

Corollary 3 presents the feasible pricing region indirectly. We can further derive the explicit price region. Using integral

$$
{ }^{6} \text { Note that } V_{q}(x, q(x))=\left.\frac{\partial V(\theta, q)}{\partial q}\right|_{\theta=x, q=q(x)}, q_{\theta}(x)=\left.\frac{\partial q(\theta)}{\partial \theta}\right|_{\theta=x} .
$$


calculus, we have:

$$
\begin{aligned}
\pi(x) & =\int_{\theta_{s}}^{x} V_{q}(\theta, q(\theta)) \cdot q_{\theta}(\theta) \mathrm{d} \theta+c \\
& =\int_{\theta_{s}}^{x} \frac{\mathrm{d} V(\theta, q(\theta))}{\mathrm{d} \theta} \mathrm{d} \theta-\int_{\theta_{s}}^{x} V_{\theta}(\theta, q(\theta)) \mathrm{d} \theta+c \\
& =V(x, q(x))-V\left(\theta_{s}, q\left(\theta_{s}\right)\right)-\int_{\theta_{s}}^{x} \frac{q(\theta)}{1+\theta q(\theta)} \mathrm{d} \theta+c
\end{aligned}
$$

where $c$ is the integral constant. According to the first condition in Corollary 3, we have $0 \leq c \leq V\left(\theta_{s}, q\left(\theta_{s}\right)\right)$.

The best prices for a feasible contract with fixed quality assignment are shown in the following corollary.

Corollary 4: Let $\mathscr{C}$ be a feasible contract with fixed quality assignment $q(\theta)$. Then the unique best price assignment for Eq. (30), denoted by $\hat{\pi}(\theta)$, are given by:

$$
\hat{\pi}(x)=V(x, q(x))-\int_{\theta_{s}}^{x} \frac{q(\theta)}{1+\theta q(\theta)} \mathrm{d} \theta
$$

In fact, as we substitute the integral constant $c$ in Eq. (36) with the its maximum allowable value, i.e., $V\left(\theta_{s}, q\left(\theta_{s}\right)\right)$, we can obtain the best price assignment Eq. (37). For example, if $q(\theta)=\theta$, the best price assignment is $\hat{\pi}(\theta)=\frac{1}{2} \log (1+$ $\left.\theta^{2}\right)+\frac{1}{2} \log \left(1+\theta_{s}^{2}\right)$. It is easy to validate that, for arbitrary $\theta \in \Theta$, the IC and IR constraints hold, i.e., $V(\theta, q(\theta))-\hat{\pi}(\theta) \geq$ $V\left(\theta, q\left(\theta^{\prime}\right)\right)-\hat{\pi}\left(\theta^{\prime}\right)$ and $V(\theta, q(\theta))-\hat{\pi}(\theta) \geq 0, \forall \theta^{\prime} \neq \theta$.

Substituting Eq. (37) for the prices in Eq. (30), we can derive the maximum utility of $\mathrm{PO}$ in a fixed quality assignment:

$$
R_{\{q(\theta)\}}^{*}=\int_{\theta_{s}}^{\theta_{e}}(\hat{\pi}(\theta)-C(q(\theta))) f(\theta) \mathrm{d} \theta
$$

where $\hat{\pi}(\theta)$ is given by Eq. (37).

Using integration by parts, we have:

$$
\begin{aligned}
& \int_{\theta_{s}}^{\theta_{e}}\left(\int_{\theta_{s}}^{\theta} \frac{q(x)}{1+x q(x)} \mathrm{d} x\right) f(\theta) \mathrm{d} \theta \\
= & \left.F(\theta) \int_{\theta_{s}}^{\theta} \frac{q(x)}{1+x q(x)} \mathrm{d} x\right|_{\theta_{s}} ^{\theta^{e}}-\int_{\theta_{s}}^{\theta_{e}} F(\theta) \frac{q(\theta)}{1+\theta q(\theta)} \mathrm{d} \theta \\
= & \int_{\theta_{s}}^{\theta_{e}}(1-F(\theta)) \cdot \frac{q(\theta)}{1+\theta q(\theta)} \mathrm{d} \theta
\end{aligned}
$$

The last line follows because $F\left(\theta_{s}\right)=0$ and $F\left(\theta_{e}\right)=1$. Thus we can rewrite Eq. (38) as follows:

$$
\begin{aligned}
& R_{\{q(\theta)\}}^{*}= \\
& \int_{\theta_{s}}^{\theta_{e}}\left(V(\theta, q(\theta))-C(q(\theta))-\frac{1-F(\theta)}{f(\theta)} \frac{q(\theta)}{1+\theta q(\theta)}\right) f(\theta) \mathrm{d} \theta
\end{aligned}
$$

It is notable that Eqs. (40) and (24) have the similar form. In fact, in a discrete model with infinite dense consumer types, the term $N_{t}$ converges to $f\left(\theta_{t}\right), \sum_{i=1}^{t} N_{i}$ and $\sum_{i=t+1}^{T} N_{i}$ converges to $F\left(\theta_{t}\right)$ and $1-F\left(\theta_{t}\right)$, respectively. Besides, $\Lambda_{t}$ converges to $-\frac{q(\theta)}{1+\theta q(\theta)}$ since $V\left(\theta_{t}, q_{t}\right)$ converges to $\int_{\theta_{s}}^{\theta_{t}} \frac{q(\theta)}{1+\theta q(\theta)} \mathrm{d} \theta$ and $V\left(\theta_{t}, q_{t}\right)-V\left(\theta_{t+1}, q_{t}\right)$ converges to $-\mathrm{d} V(\theta, q(\theta))$. Therefore, we can design the similar algorithm to computed the best quality assignment. Formally, we present the detail algorithm as follows:
1) initiate $\hat{q}(\theta)=\arg \max _{q} G(\theta, q), \forall \theta \in \Theta$

2) while $\hat{q}(\theta)$ is not feasible, do:

- find a infeasible region $[a, b] \subseteq \Theta$

- set $\hat{q}(\theta)=\arg \max _{q} \int_{a}^{b} G(\theta, q) \mathrm{d} \theta, \forall \theta \in[a, b]$

where $G(\theta, q)$ is the integrand in Eq. (40), and an infeasible region is defined as a subset of $\Theta$, say $[a, b]$, such that $q_{\theta}(\theta) \leq$ $0, \forall \theta \in[a, b]$, and $q(a)>q(b)$.

Now we consider the case that the spectrum for sale is limited, i.e., limited spectrum trading in the continue-consumertype model. Similar to that in discrete model, the PO can maximize his utility by selectively selling the channels to the SUs upon the critical type. Here the critical type is defined as a consumer type $\theta_{t_{0}}$ such that $1-F\left(\theta_{t_{0}}\right)=M^{\prime}$ where $M^{\prime}=\frac{M}{N}$ is the normalized number of channels and $N$ is the number of SUs. Then the optimal contract in limited spectrum trading in continue model can be derived by means of the similar method in discrete model, i.e., Eq. (29).

\section{Simulation Results}

We implement the proposed quality-price contract in a discrete-consumer-type model which contains $T=25 \mathrm{SU}$ types. The set of SU types is $\Theta=\{1,2, \ldots, 25\}$ and each SU's type is distributed with discrete probability distribution $z\left(\theta_{t}\right)$ on the set $\Theta$. The cost function of PO is defined as $C(q)=C_{0}+a \cdot q^{b}$ where $C_{0}=0.01, a=2$ and $b=1.2$. The lower-bound and upper-bound of the maximum allowable power are $P_{\min }=0.01$ and $P_{\max }=1$, respectively.

We study the optimal contracts in 3 system scenarios which differ from each other in the distribution of consumer types. In case (a), the larger consumer type has higher proportion (probability) than the smaller type, while in case (c) it is just the opposite. In case (b), all consumer types are uniformly distributed on the set $\Theta$. Without loss of generality, we assume that in case (a) $z\left(\theta_{t}\right)=\frac{1}{D} \theta_{t}$, (b) $z\left(\theta_{t}\right)=\frac{1}{D} \theta_{T-t+1}$, and (c) $z\left(\theta_{t}\right)=\frac{1}{T}$, where $D=\sum_{t=1}^{T} \theta_{t}$.

Figure 8 presents the quality assignments and price assignments in the optimal contracts. The stellate curves, i.e., $q(\theta)^{*}$ and $\pi(\theta)^{*}$, denote the social optimal quality assignment which maximizes the social surplus, and the related best price assignment given by Lemma 4, respectively, i.e., $q\left(\theta_{t}\right)^{*}=$ $\arg \max _{q} S\left(\theta_{t}, q\right)$ and $\pi\left(\theta_{t}\right)^{*}=V\left(\theta_{1}, q\left(\theta_{1}\right)^{*}\right)+\sum_{i=1}^{t} \Delta_{i}$. Note that the social optimal quality and price do not depend on the distribution of consumer types. The dotted curves denote the optimal quality assignments and the best price assignments in the optimal contracts for all scenarios (a), (b) and (c). Note the hollow circles denote an aborted trading process, i.e., quality $q=\mathrm{Na}$ (or price $\pi=0$ ).

From Figure 8, we find that the quality assignment in optimal contract is always less than the social optimal quality assignment, which coincides with the conclusion in Proposition 4. From economic aspect, this can be explain as follows. For the propose of revenue maximizing, the PO will reduce the qualities for the lower types SUs, so as to reduce the interest of higher type SUs on these qualities, and accordingly raise the price for the higher type SUs. For example, in case (b), by reducing the qualities of type $\theta_{10}$, the PO can assign a lower quality to type $\theta_{11}$ and meanwhile charge a much higher price from type $\theta_{11}$, comparing to the solution for the social 

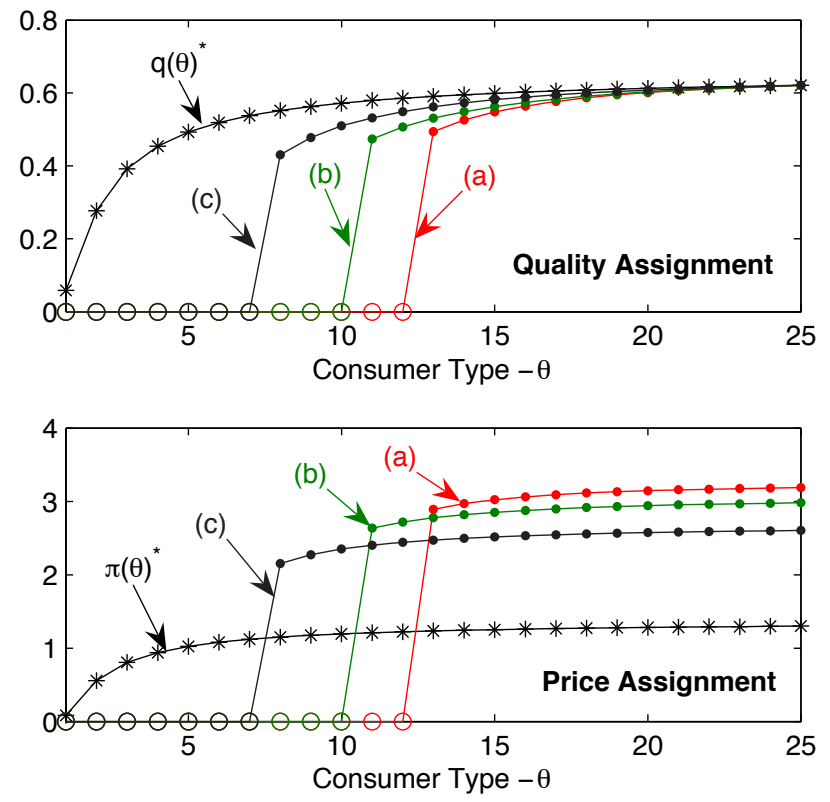

Fig. 8. The quality assignments and price assignments in the optimal contracts.

surplus maximization. Further, we find that if the number (or probability) of lower types SUs become smaller, e.g., case (a), the PO tends to reduce more qualities on the low types SUs and charge higher prices from the higher types SUs.

Figure 9 presents the social surplus and the revenues of the PO in the optimal contracts. In this bar chart, $S^{o}$ and $R^{o}$ denote the social optimal surplus, which can be obtained from $\left\{q(\theta)^{*}\right\}$, and the revenue of the PO in the social optimal quality assignment $\left\{q(\theta)^{*}\right\}$ and price assignment $\left\{\pi(\theta)^{*}\right\}$, respectively, and $S^{c}$ and $R^{c}$ denote the social surplus and the revenue of the PO under the optimal contracts. From Figure 9, we can find that a self-interested PO has no incentive to offer the social optimal quality-price assignment since the revenue $R^{o}$ is much less than $R^{c}$. The PO gains more revenue from the optimal contract, at the expense of social surplus decreasing caused by the dropping out (or qualities reducing) of low types SUs. In a practical system with limited trading spectrum, by dropping out the low type SUs, the selfish behavior of the PO will decrease the social surplus temporarily, but it may leads to a higher expected social surplus in a long-term, since the PO can assign the saving spectrum to the potential higher type SUs in the future.

Furthermore, we investigate the optimal contract in continuous-consumer-type model, which shows similar results. We also investigate the detail process of the dynamic algorithm, especially in the case of infeasible quality assignment. Due to space limitations, we do not present the detail results.

\section{CONCLUSION}

In this paper,we study the spectrum trading with single PO and multiple SUs and model the trading process as a monopoly market, in which the PO acts as monopolist and the SUs act as consumers. We design a monopolist-dominated quality-price contract, which is offered by the $\mathrm{PO}$ and consists of a set of quality-price combinations each intended for

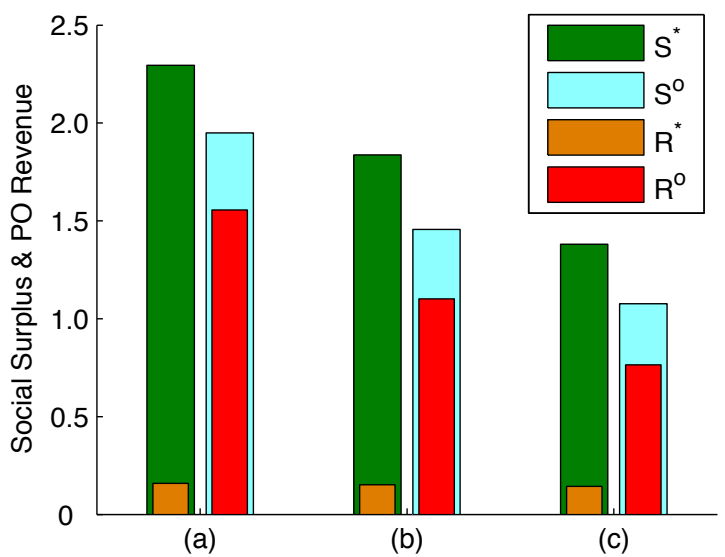

Fig. 9. The social surplus and the revenues of the $\mathrm{PO}$ in the optimal contracts.

a consumer type. We propose the necessary and sufficient conditions for the contract to be feasible. We further derive the optimal contract for both discrete-consumer-type model and continuous-consumers-type model. We analyze the social surplus, i.e., the aggregate utility of both PO and SUs, and we find that, depending on the distribution of consumer types, the social surplus under the optimal contract may be less than or close to the maximum social surplus.

\section{ACKNOWLEDGMENT}

This work is supported by National Fundamental research grant (2011CB302701, 2010CB731803，2009CB3020402), NSF China (No. 60702046, 60832005, 60972050, 60632040); China Ministry of Education (No. 20070248095); China Ministry of Education Fok Ying Tung Fund (No. 122002); Qualcomm Research Grant; China International Science and Technology Cooperation Programm (No. 2008DFA11630); PUJIANG Talents (08PJ14067); Shanghai Innovation Key Project (08511500400); National Key Project of China (2009ZX03003-006-03, 2009ZX03002-003, 2009ZX03002005); National High tech grant of China (2009AA01Z248, 2009AA1Z249, 2009AA011802); and HK RGC grants (623209, and 622410).

\section{REFERENCES}

[1] I. F. Akyildiz, W. Y. Lee, M.C. Vuran, etc., "Next Generation/Dynamic Spectrum Access/Cognitive Radio Wireless Networks: A Survey," Computer Networks, Sept. 2006.

[2] Q. Zhao and A. Swami, "A Survey of Dynamic Spectrum Access: Signal Processing and Networking Perspectives," In Proc. IEEE ICASSP '07, vol. 4, 2007.

[3] S. Haykin, "Cognitive Radio: Brain-Empowered Wireless Communications," IEEE J. Sel. Areas Commun., vol. 23(2), pp:201-220, 2005.

[4] J. Hwang and H. Yoon, "Dynamic Spectrum Management Policy for Cognitive Radio: An Analysis of Implementation Feasibility Issues," In Proc. IEEE DySPAN '08, pp:1-9, 2008.

[5] Y. Xing, C.N. Mathur, M.A. Haleem, etc., "Dynamic Spectrum Access with QoS and Interference Temperature Constraints," IEEE Trans. Mobile Comput., vol. 6, no. 4, pp:423-433, Apr. 2007.

[6] N. Nie and C. Comaniciu, "Adaptive Channel Allocation Spectrum Etiquette for Cognitive Radio Networks," In Proc. IEEE DySPAN '05, pp:269-278, Nov. 2005.

[7] J. Acharya and Roy D. Yates, "Dynamic Spectrum Allocation for Uplink Users with Heterogeneous Utilities," IEEE Trans. Wireless Commun., vol. 8, no. 3, 2009. 
[8] L. Gao, Xinbing Wang, and Y. Xu, "Multi-radio Channel Allocation in Multi-hop Wireless Networks," IEEE Trans. Mobile Comput., vol 8, no. 11, pp: 1454-1468, Nov 2009.

[9] L. Grokop and David N.C. Tse, "Spectrum Sharing between Wireless Networks," In Proc. IEEE INFOCOM '08, pp:201-205, 2008.

[10] Z. Yang, and Y. Liu, "Quality of Trilateration: Confidence based Iterative Localization," IEEE Trans. Parallel Distrib. Syst., vol. 21, no. 5, May 2010.

[11] Y. Liu, Z. Yang, etc., "Location, Localization, and Localizability," Journal of Computer Science and Technology, 25(2), Mar 2010.

[12] M. Li, and Y. Liu, "Underground Coal Mine Monitoring with Wireless Sensor Networks," ACM Trans. on Sensor Networks, 5(2), March 2009.

[13] D. Niyato and E. Hossain, "Spectrum trading in cognitive radio networks: A market-equilibrium-based approach," IEEE Wireless Commun., vol. 15 , pp:71-80, Dec. 2008

[14] D. Niyato, E. Hossain, and Z. Han, "Dynamics of Multiple-Seller and Multiple-Buyer Spectrum Trading in Cognitive Radio Networks: A Game-Theoretic Modeling Approach," IEEE Trans. Mobile Comput., 2009.

[15] C. Kloeck, H. Jaekel, and F.K. Jondral, "Dynamic and Local Combined Pricing, Allocation and Billing System with Cognitive Radios," In Proc. IEEE DySPAN '05, pp:73-81, Nov. 2005.

[16] F. Wang and M. K. Shuguang Cui, "Spectrum Sharing in Cognitive Radio Networks," In Proc. IEEE INFOCOM '08, pp:1885-1893, 2008.

[17] H. Yu, L. Gao, Z. Li. X. Wang, and E. Hossain, "Pricing for Uplink Power Control in Cognitive Radio Networks," IEEE Trans. Veh. Technol., 2010.

[18] X. Wang, Z. Li, P. Xu, Y. Xu, X. Gao, and H. Chen, "Spectrum Sharing in Cognitive Radio Networks - An Auction-based Approach," to appear in IEEE Trans. System, Man Cybern, B, Cybern., 2010.

[19] J. Huang, R. Berry, and M.L. Honig, "Auction-Based Spectrum Sharing," ACM Mobile Networks and Applications J., 11(3):405-418, 2006.

[20] L. Gao, Y. Xu, and X. Wang, "MAP: Multi-Auctioneer Progressive Auction in Dynamic Spectrum Access," to appear in IEEE Trans. Mobile Comput., 2010.

[21] Y. Xing, R. Chandramouli, and C.M. Cordeiro, "Price Dynamics in Competitive Agile Spectrum Access Markets," IEEE J. Sel. Areas Commun., vol. 25, no. 3, pp:613-621, Apr. 2007.

[22] O. Ileri, D. Samardzija, T. Sizer, etc., "Demand Responsive Pricing and Competitive Spectrum Allocation via a Spectrum Server," In Proc. IEEE DySPAN '05, pp:194-202, Nov. 2005.

[23] S. Gandhi, C. Buragohain, L. Cao, etc., "A General Framework for Wireless Spectrum Auctions," In Proc. IEEE DySPAN '07, Apr. 2007.

[24] M. A. Lariviere, "Supply chain contracting and coordination with stochastic demand," Kluwer Academic Publishers, Boston, pp:233-68.

[25] A. A. Tsay, S. Nahmias and N. Agrawal, "Modeling supply chain contracts: a review", Kluwer Academic Publishers, Boston, pp:299-336.

[26] Charles J. Corbett, D. Zhou, etc., "Designing Supply Contracts: Contract Type and Information Asymmetry," Management Sci., 50(4), 2004.

[27] A. Y. Ha, "Supplier-buyer contracting: Asymmetric cost information and cutoff level policy for buyer participation," Naval Res. Logist, 2001.

[28] Weng, Z. K., "Channel coordination and quantity discounts," Management Sci., 41(5) 1509-1522, 1995.

[29] Chen, F., A. Federgruen, etc., "Coordination mechanisms for a distribution system with one supplier and multiple retailers," Management Sci., 47(5), 2001.

[30] Spence, A.M., "Job market signaling," Quarterly Journal of Economics, $87: 355-374,1973$

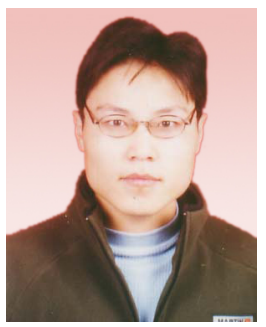

Lin Gao received the B.S. degree in Information Engineering from Nanjing University of Posts and Telecommunications, China, in 2002, and the M.S. degree in Electronic Engineering from Shanghai Jiao Tong University, China, in 2006. Currently he is pursuing his Ph.D. degree at the Institute of Wireless Communication Technology (IWCT) in Shanghai Jiao Tong University. His research interests include channel-adaptive technologies, cross-layer designs, distributed resource allocations for mobile and wireless networks, dynamic spectrum allocation and management in cognitive radio networks, game theory and learning.

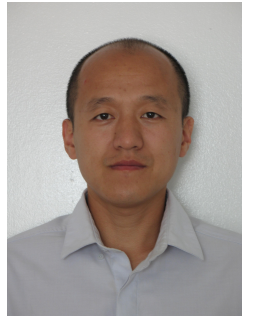

Xinbing Wang received the B.S. degree (with hons.) from the Department of Automation, Shanghai Jiaotong University, Shanghai, China, in 1998, and the M.S. degree from the Department of Computer Science and Technology, Tsinghua University, Beijing, China, in 2001. He received the Ph.D. degree, major in the Department of electrical and Computer Engineering, minor in the Department of Mathematics, North Carolina State University, Raleigh, in 2006. Currently, he is a faculty member in the Department of Electronic Engineering, Shanghai Jiaotong University, Shanghai, China. His research interests include resource allocation and management in mobile and wireless networks, TCP asymptotics analysis, wireless capacity, cross layer call admission control, asymptotics analysis of hybrid systems, and congestion control over wireless ad hoc and sensor networks. Dr.Wang has been a member of the Technical Program Committees of several conferences including IEEE INFOCOM 2009-2011, IEEE ICC 2007-2011, IEEE Globecom 2007-2011.

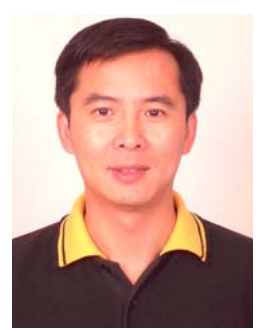

Youyun Xu was born in 1966. He was graduated from Shanghai Jiao Tong University with Ph.D. Degree in Information \& Communication Engineering in 1999. He is currently a professor with Nanjing Institute of Communication Engineering, China. He is also a part-time professor with the Institute of Wireless Communication Technology of Shanghai Jiao Tong University (SJTU), China. Dr. Xu has more than 20 years professional experience of teaching \& researching in communication theory and engineering. Now, his research interests are focusing on New Generation Wireless Mobile Communication System (IMT-Advanced \& Related), Advanced Channel Coding \& Modulation Techniques, Multiuser Information Theory \& Radio Resource Management, Wireless Sensor Networks, Cognitive Radio Networks, etc. He is a member of IEEE, a senior member of Chinese Institute of Electronics.

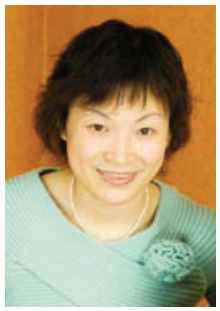

Qian Zhang received the B.S., M.S., and Ph.D. degrees from Wuhan University, China, in 1994, 1996, and 1999 , respectively, all in computer science. Dr. Zhang joined Hong Kong University of Science and Technology in Sept. 2005 where she is a full Professor in the Department of Computer Science and Engineering. She is also serving as the co-director of Huawei-HKUST innovation lab. Before that, she was in Microsoft Research, Asia, Beijing, China, from July 1999. Dr. Zhang has published more than 200 refereed papers in international leading journals and key conferences in the areas of wireless communications and networking, wireless sensor networks, wireless/Internet multimedia networking, and overlay networking. She is the inventor of about 30 pending patents.

Dr. Zhang is the Editorial Board Member of IEEE Transactions of Multimedia, IEEE Transactions on Mobile Computing, IEEE Wireless Communications Magazine, IEEE Communications Surveys and Tutorials, Elsevier Computer Communications, and Elsevier Computer Networks. Dr. Zhang is the steering committee member of IEEE Transactions of Multimedia. Dr. Zhang has received TR 100 (MIT Technology Review) world top young innovator award in 2004. She also received the Best Asia Pacific (AP) Young Researcher Award elected by IEEE Communication Society in 2004. She received the Best Paper Award in Multimedia Technical Committee (MMTC) of IEEE Communication Society and Best Paper Award for QShine 2006, Globecom 2007, ICDCS 2008, and ICC 2010. 